\title{
Mapping the cortical representation of speech sounds in a syllable repetition task
}

\author{
Christopher J. Markiewicz ${ }^{\mathrm{a}}$, Jason W. Bohland ${ }^{\mathrm{b}, *}$ \\ ${ }^{a}$ Program in Cognitive and Neural Systems, Boston University, MA 02215, USA \\ ${ }^{b}$ Department of Health Sciences, Boston University, MA 02215, USA
}

\begin{abstract}
Speech repetition relies on a series of distributed cortical representations and functional pathways. A speaker must map auditory representations of incoming sounds onto learned speech items, maintain an accurate representation of those items in short-term memory, interface that representation with the motor output system, and fluently articulate the target sequence. A "dorsal stream" consisting of posterior temporal, inferior parietal and premotor regions is thought to mediate auditory-motor representations and transformations, but the nature and activation of these representations for different portions of speech repetition tasks remains unclear. Here we mapped the correlates of phonetic and/or phonological information related to the specific phonemes and syllables that were heard, remembered, and produced using a series of cortical searchlight multi-voxel pattern analyses trained on estimates of BOLD responses from individual trials. Based on responses linked to input events (auditory syllable presentation), predictive vowel-level information was found in the left inferior frontal sulcus, while syllable prediction revealed significant clusters in the left ventral premotor cortex and central sulcus and the left mid superior temporal sulcus. Responses linked to output events (the GO signal cueing overt production) revealed strong clusters of vowel-related information bilaterally in the mid to posterior superior temporal sulcus. For the prediction of onset and coda consonants, input-linked responses yielded distributed clusters in the superior temporal cortices, which were further informative for classifiers trained on output-linked responses. Output-linked responses in the Rolandic cortex made strong predictions for the syllables and consonants produced, but their predictive power was reduced for vowels. The results of this study provide a systematic survey of how cortical response patterns covary with the identity of speech sounds, which will help to constrain and guide theoretical models of speech perception, speech production, and phonological working memory.
\end{abstract}

Keywords: speech, phonological processing, working memory, fMRI, MVPA

*Corresponding author: 635 Commonwealth Ave, Room 403, Boston MA 02215. E-mail address: jbohland@bu.edu.

Preprint submitted to NeuroImage

July 8, 2016 


\section{Introduction}

In order to perform a seemingly simple task like repeating an auditory word or syllable, our brains must rely on a series of neural representations and functional pathways. A speaker must register an auditory representation of the incoming sound sequence, map that onto learned speech items, maintain an accurate representation over any delay between the input and required output, interface that working memory representation with the motor output system, and fluently produce the target output sequence. Accordingly, to carry out this complex series of computations, the brain relies on a large, distributed set of cortical areas. While a large number of studies have sought to associate these areas with specific component processes during speech, relatively few have asked to what extent their activation patterns reflect the specific speech sounds heard, planned, or produced. The goal of the current study was to systematically map the predictive information about speech sounds at the phonemic and syllabic level available in the BOLD responses related to different components of syllable repetition. We used a delayed single syllable repetition task, which necessarily invoked phonological working memory processes, along with a sparse fMRI acquisition approach in order to provide temporal segregation of input and output related processes. This allowed us to test hypotheses about the roles of several regions thought to be involved in the encoding of speech.

The classical breakdown of speech into perceptual (i.e., for processing auditory inputs) and production (i.e., for preparing and executing motor outputs) related components (Lichtheim, 1885) has been, in part, supported by differential effects of lesions to the posterior or anterior portions of the left hemisphere. There remains considerable controversy, however, in how the brain encodes speech sounds, with some suggesting, for example that the premotor or motor cortices (traditionally associated with speech output) are automatically engaged and serve an important role in perception (Liberman et al., 1967; Fadiga and Craighero, 2003). In addition, a number of theoretical models suggest the activation of auditory cortical areas for directing speech production (i.e., target readout Guenther et al., 2006, Hickok et al., 2011). Thus, a clean segregation of circuitry between these two components is unlikely and not well supported by the existing evidence.

The theoretical framework that guides the present experiment is based on a combination of the dual streams speech processing model (Hickok and Poeppel, 2004, 2007; Rauschecker and Scott, 2009) and the GODIVA neurocomputational model (Bohland et al., 2010), which treats output-related processes of syllable sequence representation and production. A unified theory requires the specification of how speech is represented at a high level of detail across both receptive and expressive speech processes. The links between such input and output-related components are perhaps most apparent in auditory repetition, which is the focus of the present study. Repetition tasks have been suggested to rely upon the so-called dorsal stream (Hickok and Poeppel, 2007; Saur et al., 2008), a pathway which maps between auditory and motor representations of speech sounds. The dorsal stream is considered to be left hemisphere dominant, projecting from the posterior superior temporal gyrus (STg) and interconnecting the planum temporale (PT), including the region at the posterior portion of the Sylvian fissure at the parietotemporal junction (Spt), inferior parietal cortex, and premo- 
tor and inferior frontal areas. This pathway is likely to serve a critical role in repetition tasks by transforming between sensory representations and articulatory representations of speech (Saur et al. 2008). Damage to the dorsal pathway - in particular to area Spt (Buchsbaum et al. 2011) and/or to left posterior temporoparietal cortex (Baldo et al., 2012) - can result in conduction aphasia, a language disorder characterized in part by impaired repetition and problems with phonological short-term memory.

In most theoretical models of repetition, auditory inputs are mapped to motor outputs first via a phonological layer (e.g., Hartley and Houghton, 1996; Hanley et al., 2004; Nozari and Dell, 2013), and substantial work has focused on localizing such an "input buffer." At early stages of the cortical hierarchy, auditory / phonetic representations of speech inputs are supported by the posterior superior temporal lobes bilaterally (Hickok and Poeppel, 2000). Auditory association areas, which receive these inputs, then can be considered candidates for more abstract or phonological representations of a speech input sequence. The planum temporale (PT) has been shown to be functionally subdivided, with lateral portions likely involved in general auditory processing (see e.g., Binder et al., 1996) and medial portions important for processing self-produced feedback (Tremblay et al., 2013b). Furthermore, responses of anterior and middle PT have been shown to reflect the statistical structure and phonological complexity of speech sequences (Tremblay and Small, 2011, Tremblay et al., 2013a; Deschamps and Tremblay, 2014), leading to a suggested role in converting auditory inputs into phonological representations (Deschamps and Tremblay, 2014). The posterior PT has been further subdivided into lateral and medial (area Spt) portions, with the former shown to be sensitive to subsegmental manipulations in a nonword repetition task, and the latter to the number of syllables used (McGettigan et al., 2011). In their review of seven speech perception studies, Hickok and Poeppel (2007) showed that the mid to posterior STg and superior temporal sulcus (STs) respond preferentially to syllable (CV or CVC) stimuli over a variety of non-speech acoustic controls, and argued that these regions support phonological-level processes. Overt and covert speech production also induce responses in posterior STg, STs and PT ( Hickok, 2006); activation of these areas even during silent speech is consistent with a possible role in representing auditory targets for online correction of speech (Guenther et al., 2006, Hickok et al., 2011). Similarly, posterior STs responds to both heard and recalled words (Wise et al. 2001), possibly storing transient auditory representations retrieved from sensory or long-term memory.

The inferior parietal cortex (IPC) is often considered part of the dorsal stream in part due to its interconnections with superior temporal and inferior frontal regions via the arcuate fasciculus (Catani et al., 2005). The IPC contains at least seven cytoarchitectonically distinct areas Caspers et al. (2006), but most previous studies have focused on the roles of the macroanatomically defined supramarginal and angular gyri. Its importance for auditory repetition is backed by a voxel-based lesion symptom mapping (VLSM) study showing a strong association between left supramarginal gyrus (SMg) integrity and performance on word and nonword repetition tasks (Rogalsky et al., 2015). The left SMg has been commonly associated with phonological working memory (Paulesu et al., 1993), but its activation is not consistently found across speech studies. Pugh et al.(2001), in a review of previous literature, 
found both angular and supramarginal gyri to respond more to reading pseudowords than words, while a PET working memory study requiring subjects to maintain lists of words or pseudowords found no significant inferior parietal response (Fiez et al., 1996), indicating a possible sensitivity to encoding demands rather than to short-term maintenance of linguistic materials. Studies by Jonides et al. (1998) and Awh et al. (1996), on the other hand, associated IPC engagement with storage, retrieval and memory load, but not encoding. Ravizza et al. (2004) characterized dorsal and ventral IPC as being sensitive to working memory load and encoding, respectively. Thus there is general agreement that the left $\mathrm{SMg}$ and, perhaps to a lesser extent, angular gyrus are involved in phonological working memory, but the roles different IPC subregions play remain unclear. Based on the existing evidence, however, one might expect IPC, as well as the posterior STg / STs, to contain information about specific speech sounds in a syllable repetition task with strong working memory demands.

It is universally accepted that the frontal cortex is critical in directing the output of speech during repetition. Rolandic cortex is activated strongly and bilaterally during overt articulation, and with some degree of left lateralization when articulation is covert (Wildgruber et al., 1996; Riecker et al., 2000), possibly reflecting prepared articulatory commands and their predicted somatosensory consequences (Corfield et al., 1999; Lotze et al., 2000). Left lateralized activation is also seen under passive speech listening conditions with no distractor task (Wilson et al., 2004; Pulvermüller et al., 2006), and TMS priming of left motor cortex has been shown to assist phoneme discrimination in noise (D'Ausilio et al., 2009), suggesting a role for classically-defined motor output areas in some aspects of speech perception and/or perceptual judgments and their potential importance even in the input portions of repetition tasks. The adjacent ventral premotor cortex (vPMC) has been implicated in phonetic encoding specifically at the level of syllables (Peeva et al., 2010), and may function as a neural correlate of a mental syllabary (Levelt and Wheeldon, 1994) or Speech Sound Map (Guenther et al., 2006), storing sensorimotor programs for well-practiced sounds.

A phonological output buffer is a commonly proposed component in speech planning and production models (e.g., Dell et al., 1997; Roelofs, 1997; Goldrick and Rapp, 2007), and the existence of separate phonological representations for encoding speech inputs and planned outputs is supported by clinical studies (Martin et al., 1999; Jacquemot et al., 2007). The GODIVA model (Bohland et al., 2010) posits the existence of parallel phonological output buffers in the left inferior frontal sulcus (IFs) and pre-supplementary motor area (pre-SMA), with the left IFs serving to encode phonemic sequences and the pre-SMA representing abstract syllable frames (see also MacNeilage, 1998). Activation of the IFs is sensitive to both the phonological complexity of produced syllables and the complexity of planned syllable sequences (Bohland and Guenther, 2006). The IFs forms the dorsal boundary of the inferior frontal gyrus (IFg). Papoutsi et al. (2009) observed a dorsal-ventral segregation of function within IFg (see also Molnar-Szakacs et al., 2005), with the dorsal portion playing a role in phonological encoding, and the ventral portion having a more motoric role in phonetic encoding. Thus, the left IFs and/or dorsal IFg can be hypothesized to encode phonemes for output and to be engaged in the output portion of syllable repetition. As noted above, GODIVA also proposes a specific role for the pre-SMA in representing abstract syllable- 
sized frames but not phonological content. This region has similarly been proposed to be involved in lexical selection and/or premotor sequencing of speech (Tremblay and Gracco, 2006; Bohland and Guenther, 2006, Alario et al., 2006). Activation of the pre-SMA and adjacent medial premotor areas is also frequently observed in speech perception experiments without explicit production requirements, though any causal role for their activation during perception remains unclear (see also Adank, 2012).

To what extent different brain areas are explicitly involved in the encoding or representation of speech sounds is difficult to assess through traditional fMRI paradigms. This is, in large part, due to the possibility that differences in activation could be observed for a number of reasons other than a requirement to represent one or more speech sounds. Recent studies have begun to fill in existing knowledge gaps with protocols and analysis techniques that allow neural representations to be probed more explicitly. Repetition suppression (RS) paradigms have been used as an indicator that a neuronal population treats two stimuli (e.g., two instantiations of the same syllable or phoneme) as the same or different, with the assumption that trial-to-trial neuronal adaptation gives rise to suppression or enhancement of the BOLD signal. Phonological RS has implicated left posterior STg (Graves et al., 2008) in an auditory pseudoword repetition task and bilateral STs and IFg (Vaden et al., 2010) in a task requiring listening to words of with varying degrees of phonological similarity. Vaden and colleagues also reported increased activation in bilateral SMg in response to word lists with repeated phonological content, compared to word lists with purely novel phonological content (Vaden et al., 2010). Consistent with phonological representations in dorsal IFg / IFs, Myers et al. (2009) found an increased response to between-phoneme differences in voice onset time, with little sensitivity to changes within a phonetic category, of perceived syllables in this region. This was in contrast with left superior temporal regions, which showed both within- and across-category changes in activation.

Multi-voxel pattern analysis (MVPA) considers how information content across multiple voxels correlates with discrete stimulus classes (Haxby et al., 2001, Norman et al., 2006, Kriegeskorte et al., 2006). MVPA variants have been applied by a number of researchers to determine brain areas whose response patterns predict some aspect of speech stimuli (Formisano et al., 2008; Kilian-Hütten et al., 2011; Lee et al., 2012; Merrill et al., 2012, Abrams et al., 2013; Du et al., 2014; Arsenault and Buchsbaum, 2015; Correia et al., 2015; Evans and Davis, 2015; Zhang et al., 2016). Formisano et al. (2008) found responses able to discriminate between different vowels distributed bilaterally across the mid-posterior STg / STs, with responses able to discriminate different speakers of those vowels more focal and right lateralized. Using a /ba/-/da/ discrimination task, Lee et al. (2012) found that the patterns of activation in voxel clusters within the left IFg, pre-SMA, and STg were predictive of the syllable that subjects perceived, though whether these areas discriminated phonemes or syllables was unclear. In a simple listening experiment, Zhang et al. (2016) found predictive patterns for consonants and vowels, across different syllables, along the mid-STg bilaterally. Du et al. (2014) presented subjects with syllables that spanned four consonant classes under different background noise conditions. Using a multivariate method, they found that the ability for responses in different brain regions to predict the phoneme class in noise differed, with inferior frontal cortex most resilient to noise, followed by ventral 
premotor and inferior parietal cortex, with no discernibility under any noise conditions in STg. In recent work using representational similarity analysis (Kriegeskorte et al. 2008$)$, to compare the differential response patterns of local cortical areas to syllable and syllable-like acoustic inputs to those predicted by simple theoretical models, Evans and Davis (2015) have proposed a hierarchical organization of speech representations. In particular, traditional "output" areas (somatomotor cortex) appeared to have the most abstract representation of the syllable, with early auditory areas retaining the most acoustic detail. The vast majority of studies employing MVPA and related techniques have focused on speech perception rather than production; here we extend the approach to syllable repetition, which allows analysis of both input and output related representations.

In this study, we used systematically constructed CVC syllable stimuli to identify regions that correlate with phonological information at segmental and suprasegmental levels. We employed a delayed, single syllable repetition task to engage input, working memory maintenance, and output-related representations of these syllables, and a sparse fMRI paradigm to most effectively capture responses to stimulus and vocalization events. Using MVPA, we sought to localize cortical areas whose response patterns to either the input or output portions of the repetition task made significant predictions about the linguistic class labels for each stimulus. We hypothesized that MVPA analyses of the input-related responses to the auditory stimulus would highlight phonemic representations in the left posterior superior temporal sulcus and possibly Spt and/or the inferior parietal cortex. We anticipated that analyses of output-related responses would reveal a phonemic output buffer in the left inferior frontal sulcus and/or dorsal portion of the inferior frontal gyrus pars opercularis, and a syllabic representation in the left ventral premotor cortex. Because Spt has been proposed as a critical, bidirectional sensory-motor interface, we expected any predictive information in this area would also be observed during output.

\section{Materials and methods}

\subsection{Participants}

15 right-handed native American English speakers participated in this study (9 females, 6 males, mean age $=25.0, \mathrm{SD}=5.5$, range $=19-33)$. No participants reported any history of speech, language, or hearing disorders. All participants gave informed consent under the protocol approved by the Institutional Review Board of Boston University. The data from one female subject (S13) were removed from the analysis due to an abnormally high error rate during the task (see Results). For two subjects, one run (see description of sessions below) was omitted due to scanner technical problems, while two runs were omitted in a third subject. Thus, for each of 14 subjects, from 6 to 8 runs of data were analyzed.

\subsection{Task design}

Each trial began with the presentation of an auditory stimulus (syllable; see details below) and a gray fixation point, followed by an 8-9 second delay / maintenance period, after which a GO signal (the fixation point changing from gray to green) was presented, cueing participants to repeat the perceived syllable aloud. During the delay, EPI scans were 
triggered at $2 \mathrm{~s}$ and $5 \mathrm{~s}$ after the beginning of the trial. These volume acquisitions were timed to align with theoretical peak hemodynamic responses to the GO signal of the previous trial, and the stimulus presentation of the current trial, respectively (see Figure 1). The delay varied from 8 to $9 \mathrm{~s}$, in $0.1 \mathrm{~s}$ increments, to prevent participants from anticipating the GO signal.

Sessions were broken into 8 runs, each consisting of 48 trials. In 44 trials of each run, participants were presented with syllable stimuli, while control stimuli (noise) were presented in two trials, and no stimulus (silence) was presented in two trials. As a control for motor output on silence and noise trials, participants were instructed to press a button with any finger on their right hand. For two subjects, there were four noise trials and no silence trials per run.

Prior to the session, participants were informed that the stimuli they would hear were constructed from the three chosen vowels and consonants and spoken by four different talkers. They were instructed to naturally produce the syllables they heard without attempting to mimic detailed acoustic features of the specific stimulus.

\subsection{MR-data acquisition}

All measurements were performed using a 3T Philips Achieva MRI scanner with an 8 channel head coil at the Boston University Center for Biomedical Imaging. T1-weighted anatomical images were acquired for anatomical reference and coregistration with functional data $\left(0.98 \times 0.98 \times 1.2 \mathrm{~mm}^{3}\right.$ voxels, 150 sagittal slices, $256 \times 254$ matrix, repetition time $=$ $6.8 \mathrm{~ms}$, echo time $=3.1 \mathrm{~ms}, \mathrm{P}$ reduction $(\mathrm{AP}) \mathrm{SENSE}$ factor $=1.5, \mathrm{~S}$ reduction $(\mathrm{RL})$ SENSE factor $=2)$. Functional volumes consisted of 40 echo-planar transverse slices $(3 \mathrm{~mm}$ thickness), acquired in ascending order, with no gap $(3.03 \times 3.03 \times 3 \mathrm{~mm}$ voxels, $76 \times 75$ matrix, acquisition time $=2500 \mathrm{~ms}$, echo time $=35 \mathrm{~ms}$, flip angle $=90^{\circ}, \mathrm{P}$ reduction SENSE factor $=2$ ). Functional volumes were acquired in a sparse acquisition paradigm (Figure 1), triggered externally by a TTL pulse delivered from the stimulus delivery computer, mimicking a cardiac gating signal(Markiewicz, 2016). Two additional volumes were acquired at the end of each run in order to capture residual hemodynamic activity in response to previous experimental events.

Auditory stimuli were delivered through Sensimetrics S14 MRI-compatible insert earphones, and were pre-filtered to equalize the frequency response at the earphones. Subject vocalizations were recorded using an Optoacoustics FOMRI II fiber optic microphone attached to the head coil and digitized for offline analysis.

\subsection{Materials}

Stimuli were consonant-vowel-consonant (CVC) syllables designed to permit independent analysis of three segments, including a comparison of consonants in two distinct syllable positions. Stimuli were constructed from the consonants $/ \mathrm{m} /, / \mathrm{t} /$ and $/ \mathrm{l} /$ and the vowels $/ \mathrm{I} /, / \varepsilon /$ and $/ \Lambda /$ (Table 1). With the constraint that two different consonants must be used in each syllable, this produced 18 unique syllables. To select these phonemes, we parsed the CELEX English Frequency, Syllables corpus, constructed predominantly from written sources (Baayen et al., 1993), and calculated the distribution of frequencies of all 

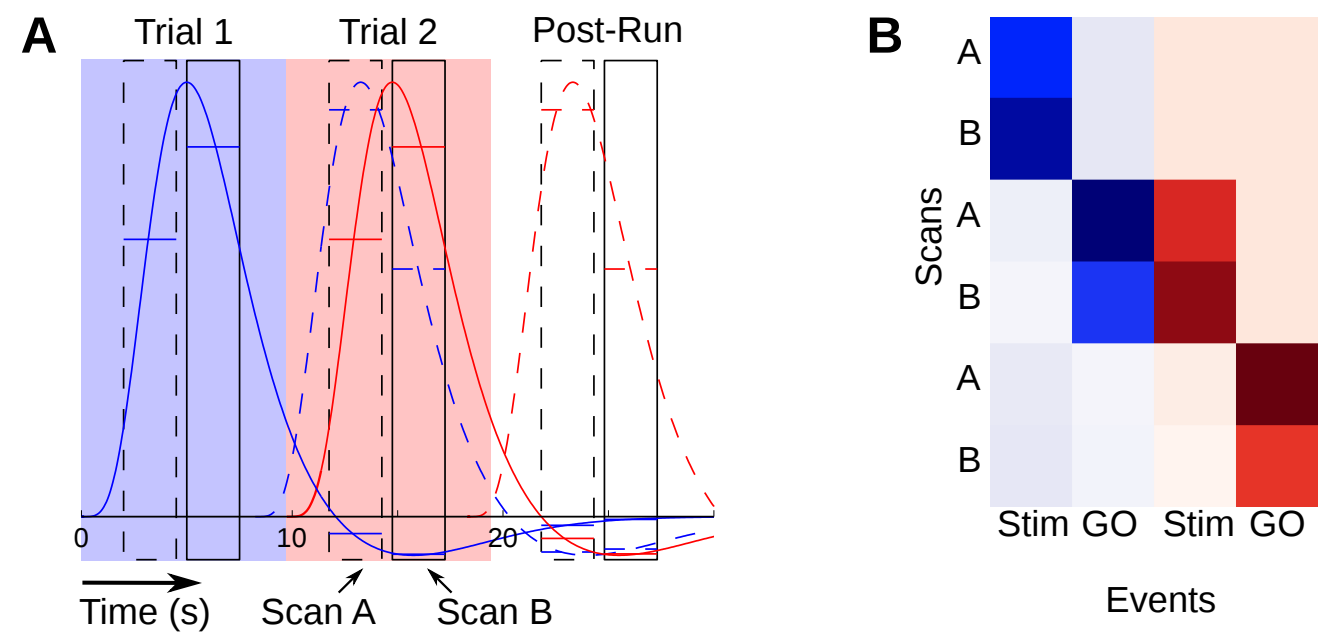

Figure 1: Specification of sparse event-related design. (A) Schema of unevenly-spaced sparse acquisition paradigm. The solid blue curve indicates the expected hemodynamic response function (HRF) associated with the presentation of the stimulus at $t=0 \mathrm{~s}$. Scans, represented by black-outlined boxes, are acquired (with $\mathrm{TA}=2500 \mathrm{~ms}$ ) beginning at $t=2 \mathrm{~s}(\mathrm{~A})$ and $t=5 \mathrm{~s}(\mathrm{~B})$. The subject is cued to produce the syllable at $t=8.5 \mathrm{~s} \pm 0.5 \mathrm{~s}$, and the dashed blue curve indicates the expected response associated with production, timed to the cue. Red curves are associated with stimulus and production cues for a second trial. Horizontal lines indicate the mean values of theoretical HRFs across the duration of a scan, which are used as regressors in a general linear model. (B) Design matrix to estimate BOLD response amplitudes to each event from the acquired scans. The blue columns depict the contributions of each scan to trial 1 events, and the red columns depict contributions to trial 2 events. See Figure 2 for further details on event estimation. 
18 CVC syllables composed of any sets of three consonants and three vowels. We chose this phoneme set to generate syllables with a wide range of frequencies of occurrence, from very infrequent ( $<1$ per million syllables) to moderately frequent (584 per million). All syllables were phonotactically legal in American English.

Two male and two female native English speakers recorded the stimuli, and five recordings of each syllable per speaker were used to allow for additional acoustic variations in the auditory tokens that subjects heard. On any given trial (see below), subjects heard a randomly selected recording of the chosen syllable, and they heard each recording from 1 to 7 times over the course of the experiment. Speech-shaped control stimuli were generated by amplitude modulating pink noise by the Hilbert envelopes of the original stimuli.

Mean formant frequencies were extracted from the approximate mid-points of vowels in both the presented stimuli and recordings of subject vocalizations using custom PRAAT (Boersma and Weenink) scripts (You et al. 2015), and random subsets were hand-checked for accuracy. Trials with vocalization formants that could not be extracted were excluded from acoustic analysis.

\begin{tabular}{|c|c|c|c|c|c|}
\hline & /I/ & \multicolumn{2}{|c|}{$\mid \varepsilon /$} & \multicolumn{2}{|c|}{$/ \Lambda /$} \\
\hline$/ \mathrm{m} /$ & $/ \mathrm{mil} /(33) \quad / \mathrm{mit} /(248)$ & $/ \mathrm{mel} /(17)$ & /met/ (298) & $/ \mathrm{m \Lambda l} /(28 / 48)$ & $/ \mathrm{m} \Lambda \mathrm{t} /(133 / 0)$ \\
\hline$/ 1 /$ & $/ \lim /(32)$ & $/ \mathrm{l} \varepsilon \mathrm{m} /(2)$ & /let/ (407) & $/ \mathrm{l}_{\Lambda \mathrm{m}} /(95 / 9)$ & $/ \mathrm{l} \Lambda \mathrm{t} /(88 / 0)$ \\
\hline$/ \mathrm{t} /$ & $/ \mathrm{t}$ Im/ (120) & $/ \mathrm{tcl} /(584)$ & $/ \mathrm{tem} /(308)$ & $/ \mathrm{t} \Lambda \mathrm{l} /(1 / 0)$ & $/ \mathrm{t}_{\Lambda \mathrm{m}} /(185 / 17)$ \\
\hline
\end{tabular}

Table 1: The consonants $/ \mathrm{m} /, / \mathrm{t} /, / \mathrm{l} /$, and vowels $/ \mathrm{I} /, / \varepsilon /, / \Lambda /$ were selected to generate CVC stimuli that span a wide range of syllable frequencies (in parentheses, per million syllables) in General American English. For the vowel $/ \Lambda /$, the first number indicates frequency of the reduced schwa vowel sound.

\subsection{Behavioral assessment}

All subjects' vocalizations were verified against the presented stimuli. Due to at times inconsistent recording quality (see Results), errors were marked only if the successfully recorded portions of vocalization were inconsistent with the stimulus. For instance, if only the sound sequence / it/ was audible, this vocalization would be considered a match to either a target of / mit/ or / lit/. Incorrect vocalizations were relabeled for analysis of output-related datasets (described below), but left unchanged for input-related analyses. Unrecognizable vowels were excluded from acoustic analysis. Verification was performed by one member of the research staff, with spot checks for ambiguous vocalizations.

\subsection{Preprocessing}

We reconstructed cortical surfaces from the MPRAGE images with FreeSurfer (Dale et al., 1999; Fischl, 2012) v5.3.0, which was also used to parcellate cortical surfaces into regions of interest according to a custom speech-centric atlas (Tourville and Guenther, 2012). All functional volumes from all runs were realigned to the first volume of the first run using the FreeSurfer Functional Analysis Stream (FsFast). A preliminary general linear model (GLM) was designed to remove linear trends and motion-related variance (using Friston's 24-parameter model Friston et al., 1996) in the BOLD signal. Regressors were entered 
separately for A and B scans, and a linear ramp was sampled at the temporal mid-points of each scan for drift removal. Finally, separate constant regressors were used to remove overall mean intensity differences between A and B scans for each run. The first volume from each run (which was not timed to a specific event of interest) in all subjects was modeled with a separate parameter and discarded. Detrending was performed using custom Python scripts.

With the exception of the estimation of overall task effects (see Results), we normalized (z-scored) the activation of each voxel relative to the mean and variance of control conditions (silence and noise trials) across all runs, prior to analysis. Control trials were thereafter excluded from analysis.

\subsubsection{Uneven scan timing correction}

To capture the peaks of both input- and output-related events, we used an unevenlyspaced sparse acquisition protocol. As a result, A and B scans are influenced by different T1 saturation effects (Schmidt et al., 2008), which is problematic since events are also intrinsically linked with different experimental conditions (i.e., input or output). To correct for this, we modeled the ratio of gray-matter intensities between B and A scans on a per-slice basis. Using naïvely motion-corrected EPI images, we calculated, across scan pairs collected from 13 subjects, the ratio of mean gray-matter intensity of B scans to A scans in each of the 40 transverse slices. These ratios are well-approximated by an exponential curve over slice index. We then applied this correction factor to A scan slices in the raw volumes and performed motion correction on the modified volumes. See Supplementary Materials A for details.

\subsubsection{Estimation of individual event responses}

We defined an input-related event as the estimated hemodynamic response to the auditory stimulus, and an output-related event as the estimated response to the initiation of the motor act (i.e., the GO signal). The specification of regressors for these events is illustrated in Figure 1. These regressors were used to construct design matrices to estimate responses to individual events from the detrended scans using a GLM as described by Perrachione and Ghosh (2013), with modifications detailed below. Acquisitions in which there was at least a half-voxel $(1.5 \mathrm{~mm})$ or greater shift from the previous volume were treated as motion outliers. Events where the magnitude of the theoretical HRF was greater than $10 \%$ of its maximum height during one of these outlier volumes were excluded from analysis. A total of 124 input-related events and 126 output-related events were excluded across subjects.

Each individual event was modeled by a canonical HRF convolved with a delta function, normalized to a maximum height of 1 (Figure 2A). For a scan that occurs during the course of the hemodynamic response, its contribution to the event was estimated as the mean of the HRF during its $2.5 \mathrm{~s}$ duration. Because a volume was captured in 40 slices, we used an HRF temporal resolution (Nipype's spm_hrf() parameter RT) of $2.5 \mathrm{~s} / 40=0.0625 \mathrm{~s}$.

The GLM estimation was performed piecewise by condition (Figure 2B), using custom Python scripts. A condition was defined as a (stimulus, event type) pair, where stimulus is a specific syllable, silence, or noise, and event type is either input-linked or output-linked. 
A

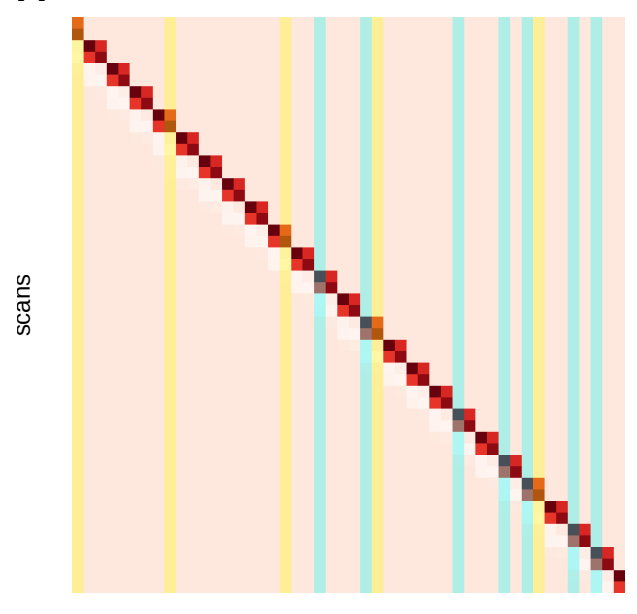

events

\section{Design matrix to estimate all events simultaneously}

B

conditions

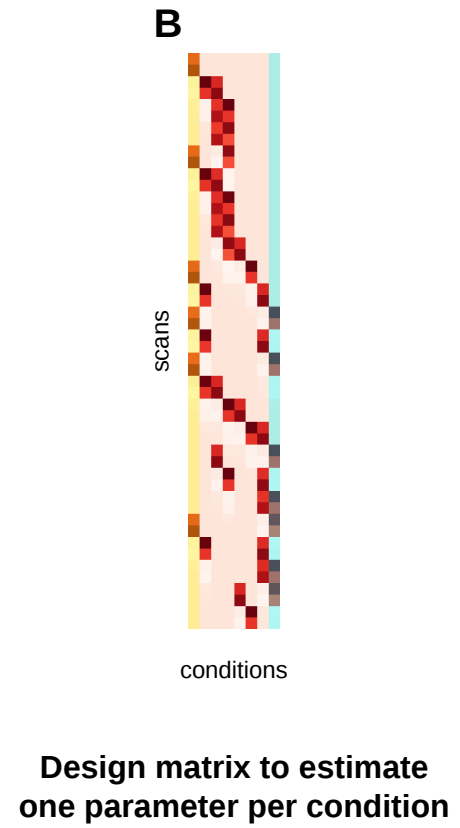

C

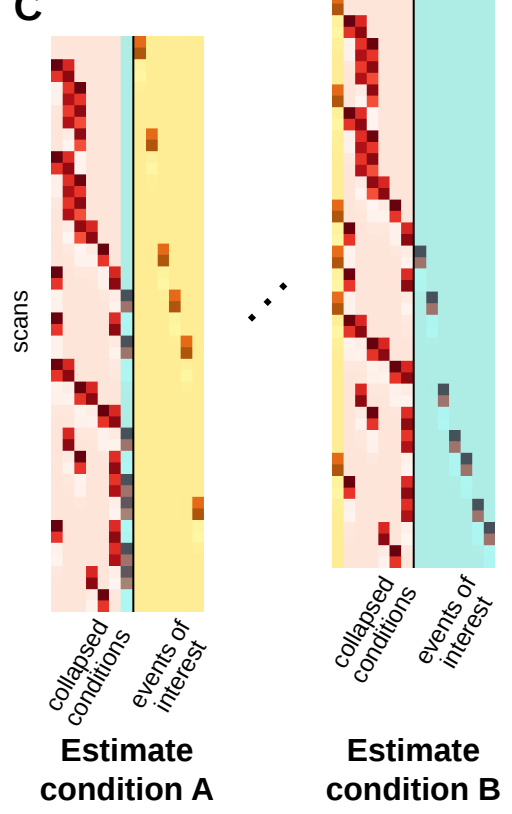

Figure 2: Piecewise general linear model. To model each event individually, events are grouped into conditions, two of which are shown in yellow and cyan in these schematized design matrices. The raw design matrix (A) contains a regressor for each event (stimulus/input or GO-signal/output), where each regressor is an impulse function convolved with an $\mathrm{HRF}$, sampled at each scan time. The condition design matrix (B) contains a regressor for each condition, which is the sum of the event-related regressors within that condition. Condition-specific design matrices (C) contain one regressor for each event in the condition of interest and one for each condition of non-interest. The estimates for the events for each condition of interest are reconstructed to produce input- and output-related datasets with one estimate per-trial. 
With 18 syllables used, this resulted in 40 conditions, between which all events may be labeled (i.e., $/ \mathrm{mel} /{ }_{\text {in }}, / \mathrm{mel} /$ out $, / \mathrm{mIl} /{ }_{\text {in }}, \ldots$... When one of these conditions was modeled, all events labeled with that condition were modeled as individual events (with individual regressors), while all other events were collapsed into a single column for each of the other conditions (Figure 2C). Only the estimates of individual events were kept, while estimates of the collapsed conditions were discarded.

An input-linked dataset for subsequent MVPA analysis was created from the HRF estimates for each input-linked event, and an output-linked dataset was created likewise. These datasets were considered separately in further analyses.

\subsection{Surface Searchlight}

We implemented a cortical surface searchlight (e.g., Chen et al., 2011; Oosterhof et al., 2011) within the PyMVPA framework (Hanke et al., 2009), using a geodesic radius of 9mm centered on each vertex of interest, calculated using Dijkstra's algorithm (Dijkstra, 1959) on the FreeSurfer mesh halfway between the pial and white matter surfaces (Chen et al.'s "graymid" surface). To avoid resampling or excess smoothing, our analyses were performed on volumetric (voxel) data in subject-native space. At a given voxel, the nearest surface vertex to the center of that voxel was selected as the center of a $9 \mathrm{~mm}$ disk, and all voxels intersecting that disk constituted the searchlight at that voxel. The result of a searchlight analysis creates an accuracy map, a volume in which each voxel contains a statistic for the searchlight centered at that voxel. For group analyses, each subject's accuracy map was projected onto their graymid surface.

The statistic of interest in our analyses was leave-one-run-out cross validation accuracy, and a linear $\mathrm{C}$ support vector machine (CSVM) was chosen as the classifier. For inputrelated analyses, the class label for each volume was the identity of the stimulus presented; for output-related datasets, the label was the identity of the speech sound produced. We performed independent searchlight analyses with full syllable $\left(C_{1} V C_{2}\right.$ trigram), onset $\left(C_{1}\right)$, vowel $(V)$, and coda $\left(C_{2}\right)$ class labels.

The PyMVPA Balancer mapper was used to ensure that, in each fold, the training and validation sets each contained identical numbers of volumes in each class, to reduce bias in SVM construction and validation. When input sets are unbalanced, the Balancer produces two random, balanced sets, each of which is classified, and the results are averaged.

\subsubsection{Nonparametric significance testing}

For each classification result, each subject's accuracy map was registered to the FreeSurfer fsaverage template for group analysis. A map of relative accuracy was created by subtracting the mean cross-validation accuracy across vertices from each vertex, such that the resulting image had a mean of zero. One-tailed $t$-tests were used to assess whether individual vertices had consistently high relative accuracy across subjects (Lee et al., 2012). The map was subjected to a $p<0.05$ (uncorrected) threshold, and we defined clusters as connected subgraphs (of supra-threshold vertices) on the surface, which were used for further inference.

To perform cluster-extent based thresholding, we generated a null distribution of chance cluster sizes. For each subject, we permuted class labels 100 times and retrained classifiers 
to generate random accuracy maps (see Stelzer et al., 2013); each such map was registered to the fsaverage template. Choosing one random accuracy map from each subject and subjecting it to the thresholding process described above produced a set of cluster sizes. In this way, we constructed $10^{4}$ sets of empirical chance cluster sizes, providing a nulldistribution for assigning cluster-level significance. Cluster-level thresholds of $p<0.01$ were used for phoneme-level analyses. A stricter threshold of $p<0.001$ was used for syllablelevel analyses; due to the lower theoretical chance-level accuracy $(1 / 18$, or $0.0 \overline{5} \%)$ random fluctuations produce a larger number of small clusters, with the effect of decreasing $p$-values for all clusters. This threshold was used to result in a minimum cluster extent that was more similar to that used for phoneme-level analysis.

\subsection{Acoustic Analysis}

Classification accuracy is driven by correlations between class labels and patterns of voxel activations. These patterns may, for instance, be consistent with a "categorical" representation, in which a voxel responds identically (modulo noise) to all stimuli drawn from the same class, or with a "continuous" representation, in which a voxel responds to an acoustic feature, which in turn correlates with class labels. Vowels, for instance, form clusters in the $F_{1}, F_{2}$ formant frequency space (Peterson and Barney, 1952$)$; a handful of voxels whose responses correlate with formants, then, could drive above-chance vowel classification.

To assess whether formant frequency related variance in the BOLD signal was driving classification accuracies, we constructed alternative design matrices for the event-estimation GLM. In these matrices, we added nuisance regressors for the first and second formants, as well as the ratio $F_{2} / F_{1}$, convolved by the HRF. With the resulting dataset, we performed an additional set of searchlight analyses, as above. Mapping into the fsaverage template and subtracting the empirical chance classification accuracy, we performed a two-sample $t$-test at each vertex between classification accuracies, where one sample contains the accuracies from the original datasets, and the other from this alternative dataset to determine if removing formant-related variance decreased classification accuracy (as would be expected if an ROI was "tracking" formant values).

\section{Results}

\subsection{Behavioral results}

One subject (S13) repeated syllables with an error rate of $20.74 \%$. For all other subjects, the mean error rate was $1.61 \%(\sigma=1.35 \%)$, with minimum and maximum error rates of 0 and $5.40 \%$. S13 was excluded from all subsequent analyses. One subject's vocalizations were not recorded for one run; however, this subject made no errors in the recorded runs, and was included in all further analyses with the assumption that no errors were made in this first run. Inconsistent recording quality resulted in 0-30 wholly or partially missing vocalizations, out of 352 total trials (mean $=13.2$, $\mathrm{std}=8.8$ ). 

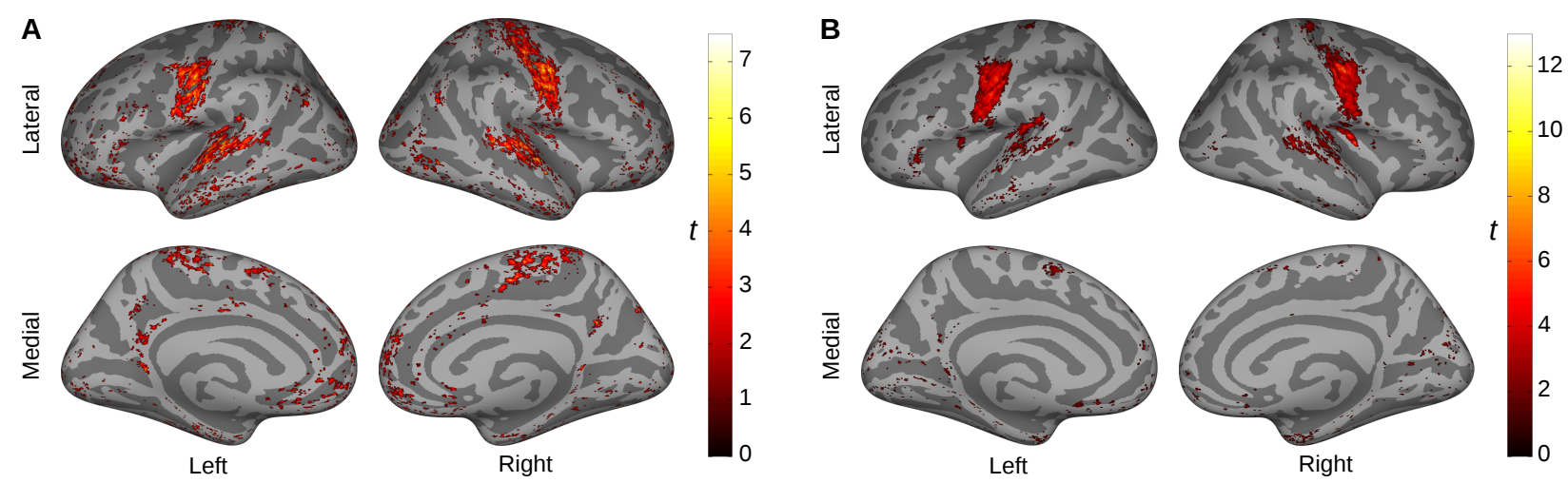

Figure 3: Overall task effects. Contrasts between mean estimates of individual event responses for task and control (silence, noise) conditions in the (A) input- and (B) output-linked hemodynamic responses. Shown are t-statistics (13 dof), thresholded at $p<0.05$ (uncorrected).

\subsection{Overall task effects}

To verify the effectiveness of individual event modeling with the piecewise general linear model, we first calculated contrasts between task and control conditions for input- and output-linked event estimates. Within each dataset, a condition is defined here as the set of estimated coefficients (betas) associated with a particular stimulus type. In this case, taskrelated (syllable responses linked to either input or output events) betas and control-related (silence or noise) betas constituted the conditions. The beta estimates for a condition were taken to be the mean of the betas for all individual events in that condition.

We contrasted the average voxel-wise betas for the task and control conditions in each subject's native space, then mapped into their FreeSurfer surface representation and onto the fsaverage template. At each vertex in the fsaverage space, we performed a two-tailed, one-sample $t$-test across participants. Figure 3 shows positive $t$-statistics that surpass an uncorrected threshold of $p<0.05$. This liberal threshold was used due to the unconventional approach of using estimates from individual events.

The input-related responses were primarily localized to bilateral superior temporal gyrus, planum temporale, and ventral motor cortex. Output-related responses were localized primarily to bilateral sensorimotor cortex, with fewer significant activations in the auditory cortex.

\subsection{Speech sound information mapping}

An MVPA searchlight analysis identifies "informative" regions with regard to the class labels assigned to a dataset, using cross-validation accuracy as the statistic of interest. Here we present results for input- and output-related datasets, analyzed with vowel, onset and coda labels, as well as with whole syllable labels. In Figures 4 and 648, the values rendered on inflated cortical surfaces are the mean raw (i.e., not normalized by the voxel-wise average) cross-validation accuracies across subjects, thresholded, using a nonparametric cluster-level significance test, with a vertex-wise uncorrected threshold of $p<0.05$ and cluster-wise thresholds of $p<0.01$ (for phoneme-level analyses) or $p<0.001$ (for syllable-level analyses). 

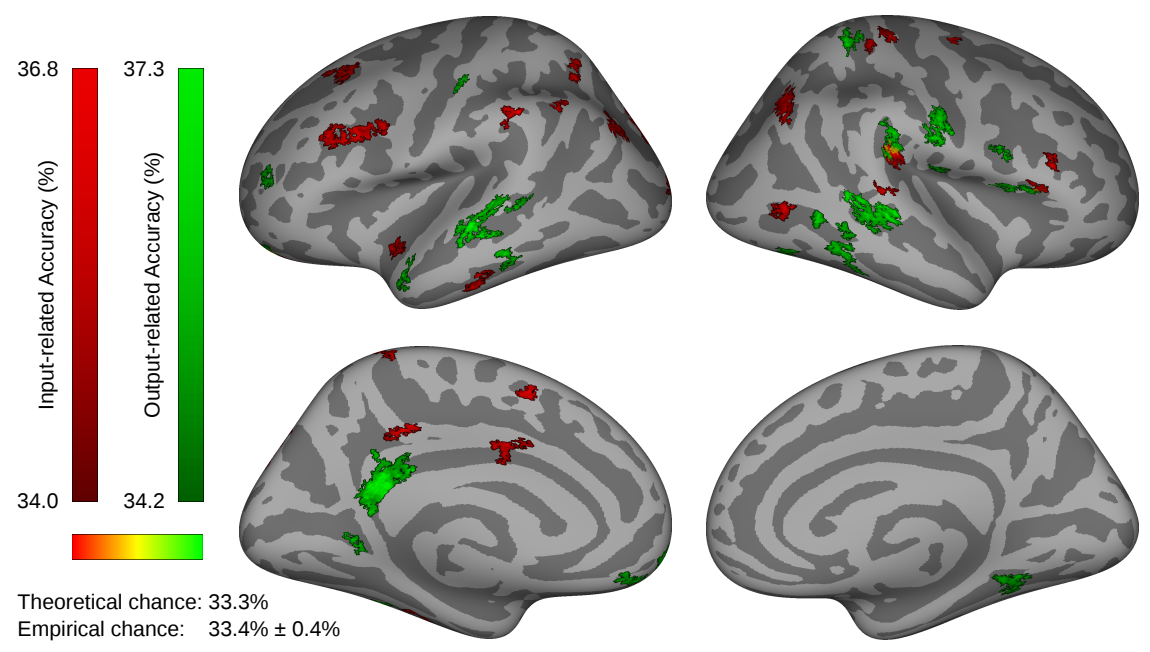

Figure 4: Vowel accuracy. Regions with significant cross-validation accuracy for decoding of vowel identity based on input- (red) and output-related (green) hemodynamic response estimates. Regions of overlap are shown in yellow, with greater input accuracy appearing more toward red and greater output accuracy appearing more toward green. For output-related analysis, the identity of the spoken vowel was used as the classification target if the vocalization differed from the presented stimulus. Results presented at $p<0.05$ uncorrected, thresholded by cluster size $(p<0.01)$.

\subsubsection{Vowels}

Figure 4 shows the mean accuracies for classifiers trained on vowel identity, irrespective 3 of the surrounding consonants, with a cluster-level threshold of $p<0.01$. This and other figures (except Figure 5) render, in different hues, areas with significant information both based on input responses and output responses, allowing a direct comparison across stages of the task. Empirical chance accuracies, indicated in Figures 4, 6, 7 and 8, represent the across-subject average and standard deviation of the spatial means of each accuracy map. For input-linked analyses, we found a superior temporal cluster centered in right posterior superior temporal gyrus (STg), in addition to inferior parietal clusters in left supramarginal gyrus (SMg), right parietal operculum (PO), and angular gyrus (AG). In addition we found a prominent cluster centered in left inferior frontal sulcus (IFs), and two smaller clusters in right IFs and inferior frontal gyrus pars opercularis (IFo). For output-linked responses, prominent clusters of predictive information were found bilaterally in posterior superior temporal sulcus (STs) and in right PO, along with inferior frontal clusters in right IFo, frontal operculum and ventral premotor cortex (vPMC). A strong, right-lateralized ventral somatosensory cortical cluster also appears, as well as a large cluster of predictive voxels in the left posterior pericallosal sulcus / cingulate gyrus.

Locations of peak classification rates for all such clusters are listed in Tables 2 and 3. Theoretical chance accuracy for classifying three vowels was $1 / 3=0 . \overline{3}$, and peak group mean accuracies ranged from 0.351 to 0.373 . Note, however, that clusters in the present analysis (and all classification analyses presented) were defined by increases over the empirical chance accuracy rate.

In order to determine the extent to which formant frequency related information was driv- 


\begin{tabular}{|c|c|c|c|c|c|c|c|c|}
\hline \multicolumn{9}{|c|}{ Input - Vowel } \\
\hline \multicolumn{3}{|c|}{ Coordinate } & Accuracy & $p$ & Extent & Hemi & aparc & SLaparc17 \\
\hline-35 & 13 & 25 & 0.362 & $2.9 \mathrm{e}-04$ & 561 & lh & caudalmiddlefrontal & pIFs \\
\hline-16 & -87 & 31 & 0.366 & $1.1 \mathrm{e}-03$ & 343 & lh & superiorparietal & $\mathrm{OC}$ \\
\hline-32 & -80 & 29 & 0.362 & $1.2 \mathrm{e}-03$ & 324 & lh & inferiorparietal & AG \\
\hline-26 & 14 & 43 & 0.361 & $1.9 \mathrm{e}-03$ & 259 & lh & caudalmiddlefrontal & pMFg \\
\hline-58 & -40 & 40 & 0.365 & $3.4 \mathrm{e}-03$ & 185 & lh & supramarginal & $\mathrm{aSMg}$ \\
\hline-3 & 0 & 33 & 0.359 & $3.9 \mathrm{e}-03$ & 170 & lh & posteriorcingulate & midCG \\
\hline-14 & -42 & 70 & 0.359 & $3.9 \mathrm{e}-03$ & 170 & lh & paracentral & $\mathrm{dSC}$ \\
\hline 1 & 0 & 24 & 0.358 & $4.6 \mathrm{e}-03$ & 154 & lh & insula & Unknown \\
\hline-6 & -35 & 44 & 0.36 & $4.7 \mathrm{e}-03$ & 151 & lh & posteriorcingulate & pCG \\
\hline-56 & -26 & -28 & 0.363 & $4.9 \mathrm{e}-03$ & 148 & lh & inferiortemporal & $\mathrm{pITg}$ \\
\hline-15 & 48 & -21 & 0.36 & $5.0 \mathrm{e}-03$ & 145 & lh & lateralorbitofrontal & $\mathrm{FP}$ \\
\hline-40 & -8 & -14 & 0.357 & $5.2 \mathrm{e}-03$ & 142 & lh & insula & PP \\
\hline-50 & -57 & 37 & 0.364 & $5.3 \mathrm{e}-03$ & 139 & lh & inferiorparietal & $\mathrm{AG}$ \\
\hline-41 & -29 & -23 & 0.359 & $6.9 \mathrm{e}-03$ & 114 & lh & fusiform & pTF \\
\hline-26 & -58 & 51 & 0.36 & $7.9 \mathrm{e}-03$ & 103 & lh & superiorparietal & SPL \\
\hline-20 & -99 & 9 & 0.356 & $8.8 \mathrm{e}-03$ & 94 & lh & lateraloccipital & $\mathrm{OC}$ \\
\hline-7 & 8 & 52 & 0.362 & $9.3 \mathrm{e}-03$ & 90 & lh & superiorfrontal & preSMA \\
\hline-27 & -58 & 45 & 0.366 & $9.8 \mathrm{e}-03$ & 86 & lh & superiorparietal & SPL \\
\hline 33 & -68 & 39 & 0.368 & $2.6 \mathrm{e}-04$ & 588 & $\mathrm{rh}$ & inferiorparietal & $\mathrm{AG}$ \\
\hline 44 & -34 & 24 & 0.363 & $6.4 \mathrm{e}-04$ & 422 & $\mathrm{rh}$ & supramarginal & $\mathrm{PO}$ \\
\hline 0 & 2 & 24 & 0.358 & $2.4 \mathrm{e}-03$ & 228 & $\mathrm{rh}$ & insula & Unknown \\
\hline 29 & -30 & 64 & 0.363 & $2.7 \mathrm{e}-03$ & 212 & $\mathrm{rh}$ & postcentral & dSC \\
\hline 43 & -64 & 2 & 0.363 & $5.8 \mathrm{e}-03$ & 130 & $\mathrm{rh}$ & lateraloccipital & MTO \\
\hline 29 & -36 & 57 & 0.361 & $7.5 \mathrm{e}-03$ & 107 & $\mathrm{rh}$ & postcentral & dSC \\
\hline 65 & -32 & 2 & 0.358 & $7.8 \mathrm{e}-03$ & 104 & $\mathrm{rh}$ & superiortemporal & pdSTs \\
\hline 50 & 22 & 7 & 0.353 & $8.3 \mathrm{e}-03$ & 99 & $\mathrm{rh}$ & parstriangularis & vIFo \\
\hline 19 & -2 & -11 & 0.351 & $9.0 \mathrm{e}-03$ & 93 & $\mathrm{rh}$ & insula & Unknown \\
\hline 47 & 27 & 18 & 0.359 & $1.0 \mathrm{e}-02$ & 85 & $\mathrm{rh}$ & parsopercularis & pIFs \\
\hline 24 & -11 & 54 & 0.36 & $1.0 \mathrm{e}-02$ & 85 & $\mathrm{rh}$ & precentral & mdPMC \\
\hline
\end{tabular}

Table 2: Significant clusters for vowel-classification in input-linked dataset. Columns: (a) Coordinates of peak accuracy, in MNI305 space; (b) mean cross-validation accuracy, across subjects; (c) empirical clusterwise $p$-value; (d) extent (\# of contiguous vertices); (e) hemisphere; (f) Desikan et al. (2006) atlas label; (g) Tourville and Guenther (2012) speech-related atlas label.

1 ing classification accuracy for vowels (the most acoustically salient segments), we trained

2 classifiers on events modeled with and without acoustic nuisance regressors specifying the

3 first and second formant frequencies (and their ratio) of vowels heard or produced. The

4 formant values extracted for all stimuli as well as a random subset of subjects' productions

5 are shown in Supplementary Materials C. Vertex-wise two-sample $t$-tests were conducted

6 between the relative vowel classification rates resulting from searchlight analyses for these

7 two models. Figure 5 shows the resulting $t$-statistics, masked by the clusters identified in

8 the input- and output-linked vowel searchlight analyses. In both input- and output-related

9 analyses, nearly all regions showed positive $t$-statistics, indicating that removing systematic

10 variations related to formants resulted in decreased classification accuracy relative to empir-

11 ical chance accuracy, in regions identified as containing information about vowel identities.

12 However, no regions reached statistical significance when applying a false discovery rate

13 correction.

\subsubsection{Consonants}

Searchlight classification using consonants as class labels (performed separately for onset and coda positions) revealed a pattern of areas largely distinct from that found for vowels. 

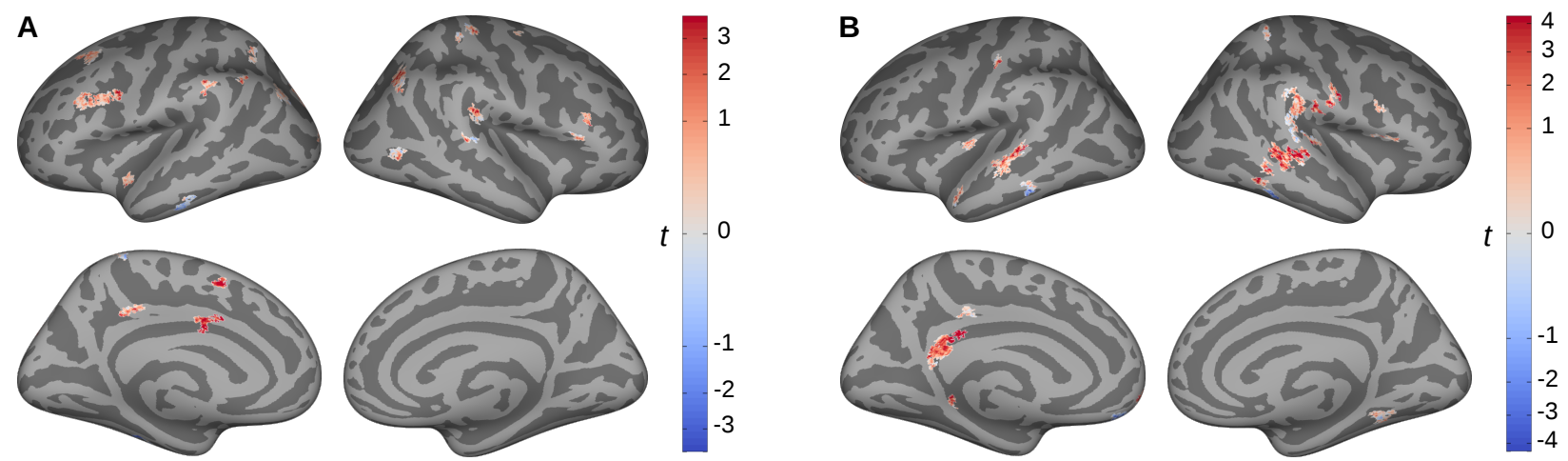

Figure 5: Differences in vowel accuracy with and without acoustic regressors. Two-sample $t$ statistics between relative vowel classification accuracies using event modeling with and without acoustic nuisance regressors, for (A) input- and (B) output-related responses. Maps are thresholded by $(p<0.01)$ cluster thresholds from the corresponding searchlight analyses (Figure44). Positive (red) values indicate superior relative classification without nuisance regressors; negative (blue) values indicate superior classification with nuisance regressors. Color axes are scaled quadratically to enhance visual separation.
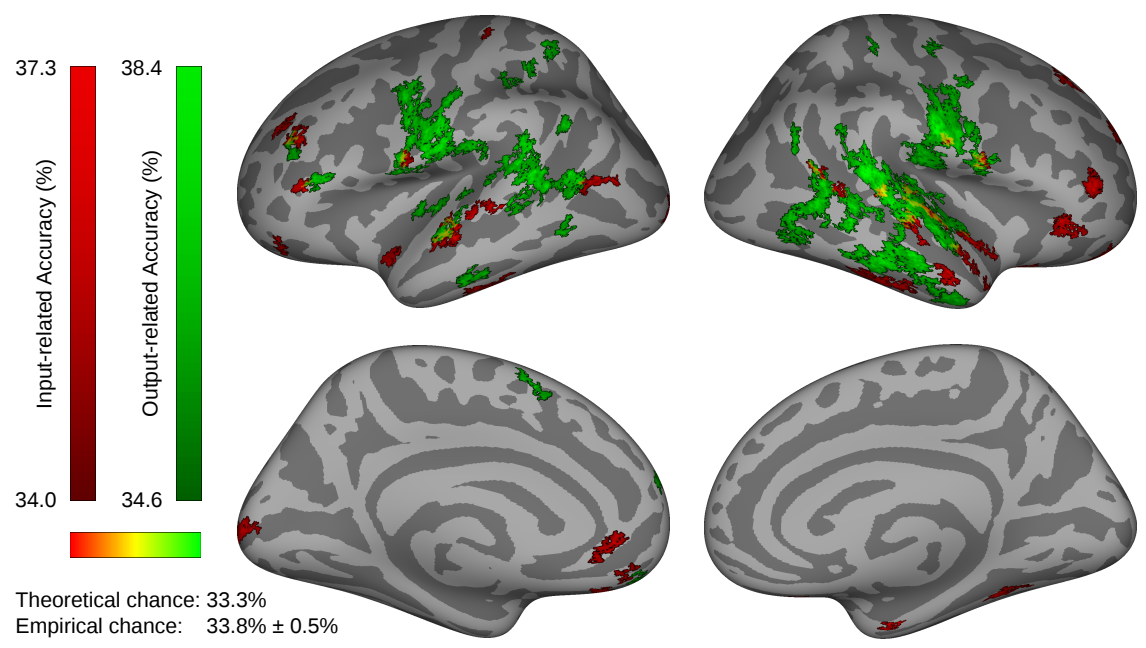

Figure 6: Onset consonant accuracy. Regions with significant cross-validation accuracy for decoding onset consonant identity based on input- (red) and output-related (green) hemodynamic response estimates. Regions of overlap are shown in yellow, with greater input accuracy appearing more red and greater output accuracy appearing more green. For output-related analysis, the identity of the spoken consonant was used as the classification target, when the vocalization differed from the presented stimulus. Results presented at $p<0.05$ uncorrected, thresholded by cluster size $(p<0.01)$. 
Output - Vowel (vocalized)

\begin{tabular}{ccc|l|c|c|c|l|l}
\hline \multicolumn{2}{c|}{ Coordinate } & Accuracy & $p$ & Extent & Hemi & aparc & SLaparc17 \\
\hline-54 & -22 & -5 & 0.373 & $6.4 \mathrm{e}-05$ & 921 & $\mathrm{lh}$ & superiortemporal & pdSTs \\
-4 & -48 & 18 & 0.37 & $7.4 \mathrm{e}-05$ & 881 & $\mathrm{lh}$ & isthmuscingulate & pCG \\
-53 & 8 & -20 & 0.363 & $3.6 \mathrm{e}-03$ & 182 & $\mathrm{lh}$ & superiortemporal & adSTs \\
-5 & 49 & -24 & 0.366 & $4.4 \mathrm{e}-03$ & 160 & $\mathrm{lh}$ & medialorbitofrontal & FP \\
-8 & 62 & -13 & 0.367 & $5.7 \mathrm{e}-03$ & 134 & $\mathrm{lh}$ & frontalpole & FP \\
-20 & -51 & -3 & 0.36 & $5.9 \mathrm{e}-03$ & 130 & $\mathrm{lh}$ & lingual & LG \\
-65 & -33 & -17 & 0.361 & $6.5 \mathrm{e}-03$ & 121 & $\mathrm{lh}$ & middletemporal & pMTg \\
-48 & -18 & 50 & 0.363 & $6.5 \mathrm{e}-03$ & 121 & $\mathrm{lh}$ & postcentral & dSC \\
-34 & 51 & 15 & 0.361 & $7.3 \mathrm{e}-03$ & 111 & $\mathrm{lh}$ & rostralmiddlefrontal & FP \\
-52 & -37 & 0 & 0.359 & $7.8 \mathrm{e}-03$ & 105 & $\mathrm{lh}$ & bankssts & pdSTs \\
-18 & 46 & -16 & 0.363 & $8.1 \mathrm{e}-03$ & 102 & $\mathrm{lh}$ & lateralorbitofrontal & FP \\
-41 & -43 & -22 & 0.366 & $8.2 \mathrm{e}-03$ & 101 & $\mathrm{lh}$ & fusiform & $\mathrm{pTF}$ \\
47 & -29 & -2 & 0.367 & $5.1 \mathrm{e}-05$ & 989 & $\mathrm{rh}$ & superiortemporal & pdSTs \\
64 & -14 & 25 & 0.369 & $1.8 \mathrm{e}-04$ & 688 & $\mathrm{rh}$ & postcentral & vSC \\
54 & -31 & 30 & 0.362 & $5.2 \mathrm{e}-04$ & 472 & $\mathrm{rh}$ & supramarginal & PO \\
29 & -53 & -6 & 0.36 & $1.6 \mathrm{e}-03$ & 290 & $\mathrm{rh}$ & lingual & LG \\
29 & -44 & 62 & 0.368 & $1.7 \mathrm{e}-03$ & 279 & $\mathrm{rh}$ & superiorparietal & SPL \\
59 & -48 & -20 & 0.365 & $2.0 \mathrm{e}-03$ & 255 & $\mathrm{rh}$ & inferiortemporal & pITg \\
39 & 9 & 10 & 0.361 & $5.6 \mathrm{e}-03$ & 136 & $\mathrm{rh}$ & parsopercularis & pFO \\
44 & -14 & 20 & 0.359 & $6.7 \mathrm{e}-03$ & 119 & $\mathrm{rh}$ & postcentral & pCO \\
40 & -67 & -16 & 0.363 & $7.4 \mathrm{e}-03$ & 110 & $\mathrm{rh}$ & fusiform & TOF \\
46 & 8 & 18 & 0.357 & $7.9 \mathrm{e}-03$ & 104 & $\mathrm{rh}$ & parsopercularis & dIFo \\
44 & 19 & 7 & 0.362 & $9.2 \mathrm{e}-03$ & 92 & $\mathrm{rh}$ & parsopercularis & vIFo \\
64 & -46 & -6 & 0.356 & $9.4 \mathrm{e}-03$ & 90 & $\mathrm{rh}$ & middletemporal & pMTg \\
56 & -54 & -3 & 0.365 & $9.4 \mathrm{e}-03$ & 90 & $\mathrm{rh}$ & middletemporal & MTO
\end{tabular}

Table 3: Significant clusters for vowel-classification in output-linked dataset. Columns: (a) Coordinates of peak accuracy, in MNI305 space; (b) mean cross-validation accuracy, across subjects; (c) empirical clusterwise $p$-value; (d) extent (\# of contiguous vertices); (e) hemisphere; (f) Desikan et al. (2006) atlas label; (g) Tourville and Guenther (2012) speech-related atlas label.

1 Figure 6 shows mean cross-validation accuracies for classifiers trained on onset identity, with 2 a cluster threshold of $p<0.01$. The input-linked analysis shows predominantly superior tem3 poral clusters, bilaterally, in addition to a few small clusters in inferior frontal regions. We 4 found predictive information in right inferior frontal gyrus (IFg) pars orbitalis and anterior 5 STg. Clusters with informative activation patterns were also found in left anterior planum 6 polare (PP), as in the vowel analysis. Further findings included superior temporal sensitivity 7 centered in STs bilaterally, in right-lateralized planum temporale (PT) and Heschl's gyrus $8(\mathrm{H})$. In inferior frontal regions, we found a left-lateralized cluster centered in IFs (anterior 9 to that observed for vowel identity) and right-lateralized vPMC.

For the corresponding output-linked analyses, ventral somatosensory and motor cortices provided strong predictive information. In superior temporal regions, we found a left posterior cluster spanning posterior $\mathrm{STs} / \mathrm{STg} / \mathrm{PP}$ and smaller clusters centered in anterior STs, Heschl's gyrus and posterior insula, as well as a cluster spanning much of the right STg, into PT/H/PP. Finally, we found a left lateralized cluster in ventral IFs/dorsal IFg pars triangularis (IFt).

Locations of peak classification rates for all such clusters are listed in Supplementary Tables B.1-B.2. Theoretical chance accuracy for classifying three consonants was again $0 . \overline{3}$, and peak group mean accuracies ranged from 0.354 to 0.384 .

We next examined the information associated with prediction of coda consonant identity. 

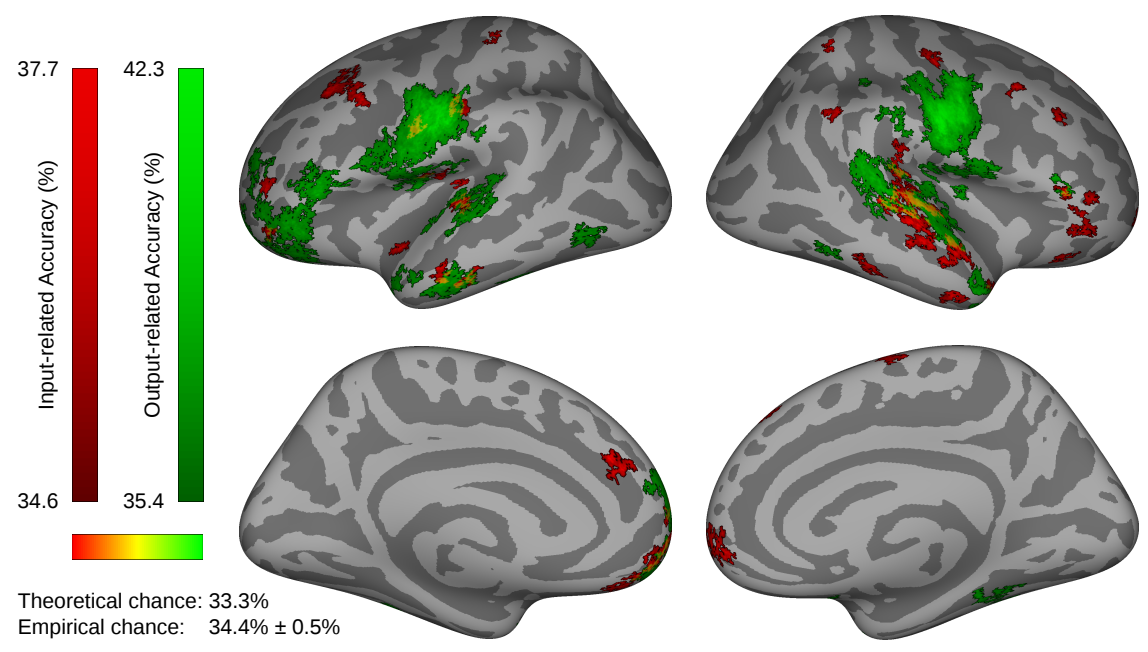

Figure 7: Coda consonant accuracy. Regions with significant cross-validation accuracy for decoding coda consonant identity based on input- (red) and output-related (green) hemodynamic response estimates. Regions of overlap are shown in yellow, with greater input accuracy appearing more red and greater output accuracy appearing more green. For output-related analysis, the identity of the spoken consonant was used as the classification target, when the vocalization differed from the presented stimulus. Results presented at $p<0.05$ uncorrected, thresholded by cluster size $(p<0.01)$.

Mean cross-validation accuracies for classifiers trained on coda identity are shown in Figure 2 7. For input-linked analyses, we found superior temporal sensitivity in left Heschl's gyrus 3 and PP, a large right-hemisphere cluster spanning mid-STs/STg, Heschl's gyrus and PT, and 4 a further cluster centered in right anterior STg. Right lateralized inferior parietal clusters 5 were found in AG and PO, and right-lateralized inferior frontal clusters were found in IFt.

For the corresponding output-linked analyses, ventral sensorimotor cortices were prominent, bilaterally, with especially high group mean classification accuracies. Again, as seen for classification of onsets, superior temporal lobe sensitivity was localized to posterior STg in the left hemisphere, and in posterior and anterior STg in the right hemisphere. Bilateral clusters were also found in SMg and IFt.

Locations of peak classification rates for all such clusters are listed in Supplementary

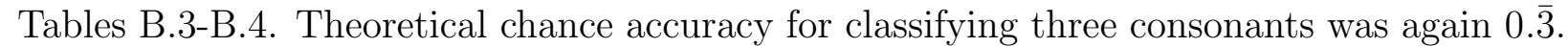
Peak group mean accuracies for the input-linked analysis ranged from 0.363 to 0.377 , while those for the output-linked analysis ranged from 0.370 to 0.423 .

\subsubsection{Syllables}

Finally, we examined the information associated with prediction of whole-syllable identity. Figure 8A shows mean cross-validation accuracies for classifiers trained on syllable identity, with a cluster-level threshold of $p<0.001$. Input-linked response estimates in left STs/STg, right anterior STg, posterior insula (pINS) and Heschl's gyrus provided significant predictive power for the syllable heard by the subject. We also found inferior frontal clusters in the left vPMC and vMC, as well as right frontal operculum and IFg pars orbitalis. For output-linked responses, predictive voxel patterns were localized in the left posterior STg, 


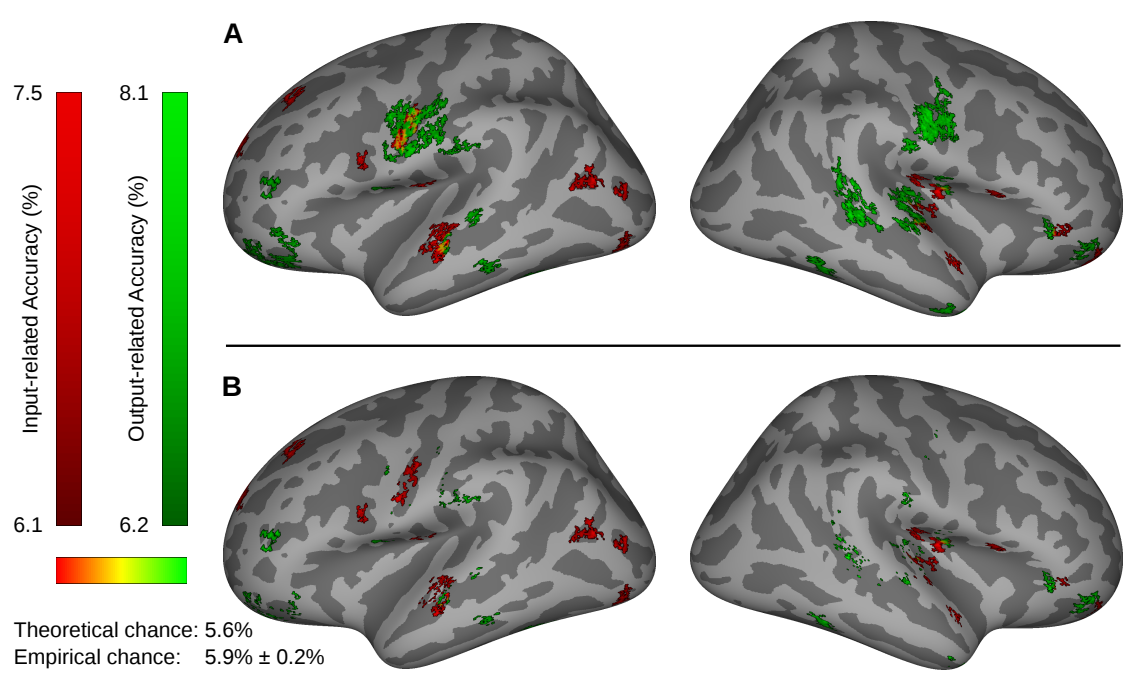

Figure 8: Syllable accuracy. (A) Regions with significant cross-validation accuracy for decoding of syllable identity based on input- (red) and output-related (green) hemodynamic response estimates. Regions of overlap are shown in yellow, with greater input accuracy appearing more red and greater output accuracy appearing more green. For output-related analysis, the identity of the spoken syllable was used as the classification target, when this differed from the presented stimulus. Results presented at $p<0.05$ uncorrected, thresholded by cluster size $(p<0.001)$. Medial surfaces reveal no significant results. (B) An additional mask has been applied, hiding decoding results in vertices where significant decoding of constituent phonemes was found. Phoneme-level clusters were thresholded at $p<0.01$, as shown in Figures 4.6 and 7. Input- and output-related masks were constructed separately.

mid-STs, and right hemisphere Heschl's gyrus and posterior STs. Bilaterally, we found large, ventral sensorimotor clusters and smaller anterior SMg clusters. No significant clusters were observed on the medial surface (not shown).

Figure $8 \mathrm{~B}$ shows the same values as in $8 \mathrm{~A}$, masked to reveal significant vertices that do not appear in onset, vowel, or coda results. Input-linked clusters remain largely unaltered in left vPMC and right pINS, while anterior superior temporal clusters bilaterally and left vMC were found to overlap with onset and coda clusters, to greater or lesser extents. All large output-linked syllable clusters were found to overlap with onset or coda clusters.

Locations of peak classification rates for all such clusters are listed in Supplementary Tables B.5-B.6. Theoretical chance accuracy for an 18 class classification problem was $1 / 18=0.0 \overline{5}$, and peak group mean accuracies ranged from 0.07 to 0.083 .

\section{Discussion}

In this study, we applied cortical surface searchlight-based MVPA to estimated BOLD responses during a simple syllable repetition task. We sought to determine brain areas whose response patterns offered significant predictive power about the specific speech sounds heard and repeated, and to dissociate responses that were linked to the stimulus (i.e., input-linked) and those associated with the vocal response (i.e., output-linked). Below, we first discuss the overall pattern of results observed relative to our initial hypotheses, comparing and contrasting areas that contained speech sound related information related to the stimulus or 
to vocal output across phoneme and syllable units. This is followed by a general summary and synthesis of results and discussion of more technical aspects of the study.

\subsection{Vowels}

We anticipated that MVPA performed on vowel identity would provide the strongest and most interpretable results. This is because vowels are more acoustically salient and have longer durations than their consonant counterparts. In addition, the full set of vowels that a listener must have the ability to represent is smaller than the set of consonants. Although we used the same number of distinct vowels and consonants (three each) in our study, the more limited vowel set in American English might be expected to result in a higher signal-to-noise ratio in vowel-related activity patterns. Therefore, we consider the results in Figure 4 to be the most indicative of the neural representation of phonemes.

We hypothesized that left inferior frontal sulcus (IFs) would show sensitivity to phonemes during the output phase of the task, based on previous work resulting in the GODIVA neurocomputational model (Bohland et al., 2010), which proposed that this region serves as a site for planning and selecting abstract sequences of phonemes (i.e., a phonological output buffer, Jacquemot et al., 2007). This region, stretching into dorsal inferior frontal gyrus (IFg) pars triangularis, has been shown to distinguish between-phoneme, but not withinphoneme, differences in voice-onset time (VOT) (Myers et al., 2009), as well as increase in activation in the planning of syllables of increasing phonological/phonetic complexity (Bohland and Guenther, 2006). Somewhat surprisingly, we discovered a large cluster of sensitivity to vowel identity in left IFs during the input phase of the task. We interpret this result as indicating that participants accessed the articulatory rehearsal and speech output systems immediately upon hearing and encoding the syllable. This strategy makes sense because participants were aware that they would need to remember and immediately speak the heard syllable upon receiving a GO signal.

We also therefore feel that it is important to revise our characterization of the "inputrelated" datasets to note that many responses that may be more appropriately associated with working memory or the maintenance of a speech plan. Phonological working memory has previously been proposed to depend on the left inferior parietal lobule (IPL) (e.g., Paulesu et al., 1993; Celsis et al., 1999, Raizada and Poldrack, 2007), and we discovered significant clusters in supramarginal gyrus (SMg) and angular gyrus (AG) for predicting vowels based on input-related responses. Raizada and Poldrack (2007) identified left SMg as performing categorical phonemic processing in a univariate analysis of responses to stimuli along the / ba/-/da/ continuum. A multivariate analysis of the same dataset, in which events were labeled with the subject's perceived category, however, failed to detect stimulus identity information in the same region (Lee et al., 2012). It is possible that the working memory demands of the current study, in comparison to Lee et al. (2012), account for the appearance of vowel information in this analysis. An additional input-related cluster in the left rostral supplementary motor area (pre-SMA) coincides with a peak activation likelihood estimate (ALE) found in a meta-analysis contrasting 189 working memory tasks (a subset of which were phonological tasks) against control conditions (Rottschy et al., 2012). The left IFs and 
SMg clusters observed here also lie near peaks in the "working memory network" identified by that same contrast.

We also predicted that the left posterior superior temporal sulcus (STs) would provide phonological information during the input phase of the task, and instead found bilateral posterior STs clusters during the output phase. A strong residual auditory response to the stimulus is unlikely, as the "output-related" hemodynamic response peaks approximately 13 seconds after stimulus presentation. This information, we believe, is more likely to represent some form of auditory target (e.g., Guenther et al., 2006; Tourville et al., 2008) for the acoustically salient vowel portion of the spoken syllable. A lack of input-related information may indicate that any categorical vowel representations are absent from or not strongly activated in the STs in the early stage of this specific task, or such representations are insufficiently distinct at the spatial resolution of this study.

It is notable that clusters that had predictive vowel information for the input- and outputlinked response estimates are largely non-overlapping (see Figure 4). One common cluster, however, was observed near the right hemisphere homolog of area Spt, but not in the left hemisphere. Spt has been proposed to serve as a critical auditory-motor interface (Hickok et al., 2011), so its role in predicting vowel identity in both phases of the task supports this view. The present result is also consistent with a proposed role of the right planum temporale in converting auditory inputs into phonological representations (Deschamps and Tremblay, 2014). The rightward lateralization of this cluster (and the bilateral localization of STs clusters) might reflect a right-hemisphere preference for vowel processing (Britton et al., 2009).

A number of areas that predicted vowel identity during either the input or output portions of the task were not anticipated. Based on the GODIVA model (Bohland et al., 2010), which builds upon the frame-content theory of speech production (MacNeilage, 1998), the pre-SMA, which had input-linked vowel information, is suggested to encode abstract syllabic frames without regard for phonemic content. Jonas (1981) and Ziegler et al. (1997) each concluded, based primarily on clinical studies, that the medial premotor areas are unlikely to code for specific speech sounds, but rather may be involved more generally in the sequencing and initiation of speech. For the stimuli in our study, all CVC and sequencing demands were alike. However, a number of studies have identified pre-SMA as a site of response selection in sentence, word and nonspeech facial gestures (Alario et al., 2006, Tremblay and Gracco, 2006, 2009, 2010; Tremblay and Small, 2011), and Carreiras et al. (2006, 2009) previously found differential pre-SMA response to high- and low-frequency words. Additionally, Adank (2012) has shown, based on ALE meta-analyses, that pre-SMA activity increases with the difficulty of speech comprehension tasks. If the difficulty of correctly perceiving, encoding, or selecting each syllable in phonological working memory differed across vowel classes, then this could be driving the above-chance accuracy in pre-SMA. Further study is warranted to more directly address these issues.

A surprisingly prominent right ventral angular gyrus (AG) cluster was also observed in the input-linked analysis. Left AG has classically been associated with grapheme-phoneme correspondences (Hynd and Hynd, 1984), and recent studies suggest that right AG may have a role in orthographic comprehension (Mei et al., 2014) and phonological / orthographic 
mappings (Bonte et al., 2014). We speculate that if the right AG provides information about vowel identity, it may be supported by orthographic associations with the vowels in the stimuli, although participants did not report using visual strategies in this task. Based on a thorough review of neuroimaging literature, Price $(2012)$ assigned bilateral angular gyrus a role in semantic processing, but noted that descriptions of this region's role in comprehension are still necessarily vague and insufficient. We note here that the semantic content available in syllables used was not uniform across vowels (6 of 6 /I/ syllables, 4 of $6 / \varepsilon /$ and 1 of $6 / \Lambda /$ syllables could be perceived as words or names). It is conceivable that semantically-related processes were selectively evoked for these stimuli, resulting in a signal whose discriminability artifactually correlated with vowel identity.

The large locus for output-related vowel information observed in left posterior cingulate (pCG) was unexpected since this region is not traditionally associated with speech or language. Functional connectivity studies have associated both pCG and bilateral STg with the default mode network (Martuzzi et al., 2010), which may provide a functional pathway for speech sound related information. It should be noted, however, that significant vowelrelated information was detected in bilateral STs, but not STg, for output-linked responses. Myers (2007) showed a posterior cingulate response to category-atypical VOT in a phonetic discrimination task, while Menenti et al. (2012) found a repetition suppression effect for words, as opposed to semantic or syntactic structure. Finally, working memory tasks using visually presented letters have also yielded effects in the posterior cingulate, including decreased activation with load (Tomasi et al., 2007) and activation while selecting an action among remembered targets (Hester et al., 2007). Taken together, these findings suggest a somewhat general role for posterior cingulate / precuneus in tasks requiring linguistic processing and/or working memory; the specific role of this region in our task is unclear and warrants for further investigation.

\subsubsection{Acoustic analysis: phonetic or phonological representations?}

Interpretation of MVPA studies remains an open problem, particularly because results may diverge from traditional univariate analyses, and classification success may be driven by many factors (Etzel et al., 2013, Davis and Poldrack, 2013; Todd et al., 2013; Davis et al., 2014). Our efforts to localize information corresponding to phonemes provides a window into this problem. In the case of vowels, there is a strong relationship between formant frequencies - acoustic properties of the speech sound that can vary continuously - and the identity of the vowel. Classification rates provide no obvious way to distinguish a classification based upon a neural representation of formants (an acoustic-phonetic representation) or a more abstract (phonological) representation of the vowel. We therefore tested the effect on vowel classification accuracy of removing univariate, voxel-wise BOLD responses that covary with formant frequencies specific to the sound heard or produced (see Figure 5). The idea was that, if an area maintains an abstract, categorical code for speech sounds, its classification accuracy should not be reduced by removing formant-related variance. If an area's response is instead sensitive to formant frequencies of the sound, then its basis for classifying vowel sounds may be diminished by removing such variance.

While no results reached group-level statistical significance, our findings suggest there 
may be a small decrease in classification performance when formant-related information is removed from each voxel. In particular, the pre-SMA, cingulate and posterior portion of the IFs clusters showed the strongest effects in our input-related analysis, while left posterior cingulate and right posterior STs showed the strongest effects in our output-related analysis. Areas that showed less reduction included left anterior IFs, insula, SMg, right posterior STg and IFg in input-related analysis, as well as left inferior temporal sulcus, central operculum, and right posterior STg/area Spt. The relative lack of reduction in accuracy would indicate representations that may be more abstract or phonological, as opposed to areas tracking phonetic/acoustic/articulatory details. Further work using tools such as representational similarity analysis (Kriegeskorte et al., 2008) may help to refine our understanding of the nature of these representations (see, for example, its use by Evans and Davis, 2015, to test between different levels of representation in speech perception).

\subsection{Consonants}

Consonants are acoustically and motorically dissimilar to vowels, and so provide an opportunity to observe the classification of phonemes whose acoustic and phonetic representations can be expected to substantially differ from those of vowels. Accordingly, the accuracy maps produced by classifying trials by the consonants in each stimulus (Figures 6 and 7) differ greatly from those produced by classifying by vowels. It should be noted that, unlike other studies that used stimuli with the same vowel but a consonant contrast (e.g., Myers et al., 2009; Lee et al., 2012), classifiers here had to generalize across vowel contexts to be successful (see also Zhang et al., 2016).

The accuracy maps for onset and coda consonants are broadly similar. Most prominently, information predictive of both onset and coda consonants was present bilaterally in large segments of the ventral somatosensory (vSC) and motor cortices (vMC) in output-related responses. The distinct articulations required for each consonant are the most likely source of information detected here. Notably, we did not observe similar output-related results for vowels. Articulatory (and incoming somatosensory) information corresponding to different vowels may be more difficult to differentiate because of dependence on the surrounding consonants (i.e., coarticulatory effects on vowel production), and because vowels differ more subtly in precise tongue placement (cf. different places of articulation for consonants). Such subtle variations in articulations are likely to result in less distinguishable signals for vowels in primary motor and somatosensory cortices, particularly with the current resolution of fMRI data.

Input-related analyses revealed much smaller clusters of consonant-level information in bilateral motor and premotor cortices than the corresponding output-related analyses. In the basic contrasts of both input and output event estimates vs. baseline (Figure 3), each showed strong, bilateral responses in vMC, though, though the output signal was stronger. Thus, it appears the motor / premotor cortex was engaged in the input-portion of the task, but that activation patterns were overall less predictive of consonant identity than during output, when explicit motor programs were enacted. The overlap between input and output related consonant predictors along the sensorimotor cortex is relatively small but notable. 
Though precise localization differed slightly for onsets and codas, both input- and outputrelated responses in small portions of the ventral precentral gyrus and/or sulcus, near the border of motor and premotor cortices, predicted consonant identity. These clusters appear to be consistent with the suggestion of an abstract, non-acoustic coding of consonant sounds in the ventral somatomotor regions (Evans and Davis, 2015).

Consonant information was also found broadly in the temporal cortex, bilaterally, where vowel information was more spatially confined, particularly to the superior temporal sulcus (Figure 4). Predictive clusters based on input and output event estimates overlapped mainly in the superior temporal gyrus, with left anterior STg and right middle to posterior STg clusters for onsets, and bilateral mid STg clusters for coda consonants. The wide profile of predictive responses across the superior temporal areas is difficult to interpret, but does not suggest a single area in the STg that encodes consonants in an abstract manner.

Although the onset and coda accuracy maps are broadly similar, a number of differences suggest that information relevant to consonant identity may not be represented independent of position within a syllable. However, it is not possible to distinguish between the effect of serial position on representation and the effects of primacy/recency on BOLD response in this analysis. Additional experiments will be required to elucidate important issues related to serial order within syllables, words, and sentences.

We also note that, while we attempted to minimize head movements and included regressors to account for motion in event estimates, it seems possible that some of the large, diffuse output-related results for consonants (particularly in the inferior temporal and ventral frontal pole areas) might be artifactually related to small movements. Since subjects were overtly articulating, it is possible that some consonants (especially final consonants) led to small motion-related artifacts that drove the discrimination of consonants.

\subsection{Syllables}

In addition to phoneme-level analyses, this study design permits us to discover regions whose responses correlate with the identity of the whole syllable (see Figure 8). Some findings, most obviously ventral Rolandic cortex in output-related responses, recapitulate the findings of the consonant analyses. However, a number of clusters are distinct from any of those found in the phoneme-level analyses (Figure 8B), and may indicate a representation that cannot be decomposed into smaller units.

We hypothesized that the left ventral premotor cortex would encode a syllabic representation at the time of speech output based on its proposed role as a Speech Sound Map (Guenther et al., 2006). A cluster in this region was shown to significantly predict the syllable heard, but clusters that predicted the syllable based on output-related events were more posterior in and around the central sulcus (and overlapped with consonant predictors). As was the case with left IFs for vowels, we suspect that the fact that this cluster was inputrelated is likely due to the specific task requirements, which may have allowed subjects to activate a syllabic program for speech output immediately upon hearing the stimulus. It is important to note that left vPMC did not show phoneme-level predictive information for consonants or vowels in the present study; furthermore this region was the only region 
suggested to encode entire planned syllables in a repetition suppression study conducted by Peeva et al. (2010).

Additional input-related clusters that predicted syllable identity but did not predict vowels or consonants were found in the left motor cortex along the ventral central sulcus. Recent work by Evans and Davis (2015) found left vMC to be sensitive to syllable identity, phonemic content and phoneme ordering (CV vs VC) in a speech perception task, but not to acoustic variations, such as speaker identity and acoustic degradation. We can corroborate, but not fully replicate, this finding with the vMC cluster found here; phoneme content and their order naturally support syllable classification, and the inter- and intra-speaker variations in our stimuli require some degree of abstraction from acoustic detail, but we did not present acoustically degraded stimuli.

Although additional small, distributed clusters remain for both input- and output-related prediction of syllables (Figure 8B), due to their relative size and proximity to phoneme-level clusters, we reserve the possibility that significant syllable classifications may be supported by phoneme-level information.

It is worth noting the absence of syllable-level information in the SMA/pre-SMA (or on the medial surface altogether), in the context of frame-content theory (MacNeilage, 1998) and the specific proposals of the GODIVA model (Bohland et al., 2010). In GODIVA, for CVC syllables such as those in this task, the frame representation in pre-SMA would consist of "instructions" to select a consonant, then a vowel, and then a consonant, while the phonemes themselves are encoded in left IFs. Classification for syllable identity detects response patterns that are sufficiently different between classes. If pre-SMA encodes abstract frames without content, then we would not expect predictive information to be present. Thus, this lack of syllable-level results is consistent with GODIVA predictions.

\subsection{Summary of main hypotheses}

One of our major hypotheses was that output-related responses in the left inferior frontal sulcus would provide significant information about phonemic identity, based on our suggestion that this region serves as a phonological output buffer. We found that left IFs patterns predicted vowel (but not onset or coda consonant) identity during the input portion of the syllable repetition task, suggesting perhaps the automatic recruitment of working memory and output-planning representations upon hearing the stimulus. This area did not predict the whole syllable, and thus our results support a role in phonological content at a subsyllabic level. The lack of predictive information for consonants was unexpected, but may reflect their reduced salience, increased competition due to a larger consonant alphabet compared to vowels, or a finer-grained representation that could not be recovered at the present fMRI resolution.

We also predicted that activity in the left ventral premotor cortex would predict whole syllable identity, based on the idea that this region stores sensory-motor programs for welllearned syllables or words. Indeed, a cluster in left vPMC contained significant information about whole syllable identity but not about individual phonemes. This cluster, like IFs however, appeared for input-linked rather than output-linked responses. 
On the other hand, bilateral superior temporal sulcus patterns were found to be predictive for output-related classification of vowels. We had anticipated, based on previous studies and the dual pathways model, that this region would encode phonemic content related to the auditory stimulus, but no such effect was observed. Instead, one interpretation of our results is that the strong STs clusters observed for vowel prediction reflect the activation of speech sound targets for production.

Finally, based on the dual pathways model and previous related studies, we hypothesized that area Spt and/or other portions of the planum temporale, would predict speech sounds during both input and output components of the task. This was, to an extent, confirmed, by overlapping clusters that predicted vowel identity at the two different task periods in the right hemisphere posterior Sylvian fissure.

Overall the pattern of results supported the main areas we hypothesized to be involved in representation of speech content, though our assumptions about separation of input- and output-related representations may need to be revised. A follow-up study is in progress designed to better enable separation of these representations.

\subsection{Methodological considerations}

\subsubsection{Delayed syllable repetition task}

The design of the delayed repetition task used here afforded several key features for this study. By using multiple recordings of multiple speakers, we increase ecological validity over synthesized stimuli, which are often used in categorization and discrimination studies. The naturally-occurring variation in the presented speech sounds required participants to map many acoustic stimuli onto their learned (and presumably categorical / abstract) internal memory representations, and ultimately their own motor programs for a given syllable. Further, a delayed repetition task is well-suited for examining both input- and output-related responses to the same stimuli, requiring both perception and production while allowing some temporal separation between the task components. Most previous fMRI tasks have focused on either perceptual or production processes, and our study represents, to our knowledge, the first effort to map predictive information related to speech sounds across both types of processes in the same experiment. The task shares some similarities with nonword repetition (NWR) tasks that have been used in clinical neuropsychology to assess phonological working memory in developing children and patients with aphasia (e.g., Gathercole, 1995; Jefferies et al., 2006), and to a lesser extent in neuroimaging studies with healthy subjects (McGettigan et al., 2011). In contrast to NWR, which typically uses multi-syllabic nonwords, our task used single syllable stimuli, which allowed for clear identification of sounds of interest for classification on each trial.

\subsubsection{Sparse design and hemodynamic modeling}

The experimental protocol was designed to allow as many trials as possible in order to provide sufficient training data for classifiers trained on multiple linguistic classes of interest. For this reason, we used a fast, event-related design with sparse volume acquisition (Perrachione and Ghosh, 2013), which allowed the subject to both hear and produce the stimulus during periods of relative quiet (Eden et al., 1999: Edmister et al., 1999; Hall et al., 
1999). The decision to place both volume acquisitions during the maintenance phase of each trial, such that they were timed to capture the peak response to the stimulus onset and the GO signal (from the previous trial), resulted in a pattern of unevenly timed sparse acquisitions. As a result, the single-shot EPI volumes were taken at different points on the T1 relaxation curve, and thus there were distinct slice-timing effects between A scans and B scans.

In this sparse, aperiodic paradigm, each volume acquired is inherently linked to a different event of interest, in contrast to clustered volume acquisition (CVA) designs (e.g., Zaehle et al., 2007; Schmidt et al., 2008), in which multiple volumes are acquired (sequentially) for each event of interest to reduce noise. As a consequence, HRF modeling was necessary to separate the overlapping signals. The correction described in the methods and detailed in Supplementary Materials A results in A scans with the same approximate slice-timing effects as B scans, yielding similar mean amplitudes across all trials. This permitted us to use both A and B scan data together in general linear models to estimate response amplitudes for both input- and output-related events.

\subsubsection{Input- and output-related response estimates}

The piecewise-by-condition GLMs used to estimate individual event responses (following the modeling described above) drew inspiration from the method presented by Mumford et al. (2012) to reduce collinearity in design matrices for rapid, event-related designs. In their method, an $n$-column design matrix was converted into $n 2$-column design matrices, where the second column is the sum of all other columns of the original matrix. One way to interpret this method is that all events are assumed to have an equal mean response, and variation in individual responses is treated as error; each GLM then estimates the variation attributable to the given individual event. Here we required response estimates for individual events, which were used to train and test classifiers. Our approach was to constrain all but one condition at a time (i.e., collapsing all events of each other type into single columns in the design matrix), and let the parameter estimates for all individual events in the condition of interest vary (see Figure 2).

The estimates of individual event responses were averaged and contrasted with baseline response estimates to provide basic univariate speech contrasts (Figure 3). These contrasts reveal increased hemodynamic responses primarily localized to superior temporal and ventral sensorimotor cortices, with a relative shift from auditory to somatosensory cortex in the output-linked contrast (Figure 3B). In addition to expected activations in auditory cortex when hearing a syllable, listening to speech also activates motor and premotor areas (Wilson et al., 2004; Pulvermüller et al., 2006; Meister et al., 2007; D'Ausilio et al., 2009), as was observed here. It is noteworthy, though, that despite the large cluster of input-related activation in this basic contrast, only small portions of this cluster significantly predicted the identity of phonemes or syllables, highlighting the differences between analytic approaches. Furthermore, as is observed here, output-linked events could be expected to show an overall stronger motor response and a moderate, self-induced suppression of the auditory responses to hearing one's own voice (Numminen et al., 1999; Houde et al., 2002; Flinker et al., 2010). These overall task effect maps thus provide reason for confidence in the individual event 
estimates, despite the fast temporal structure used in our protocol.

\subsubsection{Multi-voxel response patterns}

In turn, the individual event estimates form the basis for the multi-voxel pattern analyses that formed the core of this study. The MVPA approach affords certain advantages over univariate approaches. Because we were interested in responses that predict multiple features embedded within syllables, we expected that the noise introduced by the features of non-interest (e.g., all combinations of vowel and coda segments) would likely dominate the single-voxel responses to the feature of interest (e.g., /t/ in the onset position) in the analyses presented here. Using multiple voxels affords greater degrees of freedom for revealing associated response patterns. Furthermore, we would not expect individually informative voxels to align precisely across subjects; searchlight techniques introduce an implicit smoothing in the results of the analysis, facilitating greater inter-subject alignment.

In contrast to using individual event estimates, many MVPA studies (e.g., Chen et al., 2011; Oosterhof et al., 2011) use a single response estimate per-condition, per-run as the basis of classification. In so doing, these studies treat the variation from each trial as noise, and classification reflects the consistency of the mean response across runs to classes of events. Per-event classification instead treats the variation from each trial as signal, and classification reflects the generalization across distributions of responses to classes of events. This latter strategy produces cross-validation accuracies that tend to center around theoretical chance (i.e. 0.33 for 3 classes or 0.056 for 18), which are lower than commonly reported (for many stimulus classes) for the former strategy. To avoid assumptions about "chance" accuracy, and to account for spatial variations in signal, we used computationally intensive non-parametric tests to determine whether accuracies were significantly above empirical chance. It should be noted that the goal of our approach is not to "read minds," but to detect predictive information about speech sounds. Therefore, while the neural representations of speech sounds are almost certainly at a finer scale than fMRI currently affords, limiting possible classifier accuracy, statistically significant (but otherwise unimpressive) cross-validation accuracies provide such a measure of class-specific information.

\subsection{Limitations and future directions}

The stimuli used were all discrete CVC syllables, with variation derived from multiple recordings and speakers, which is in contrast with a number of studies that instead used finely varying synthetic stimuli, which may more directly allow addressing questions related to categorical processing of speech. However, such stimuli could easily be employed within a repetition paradigm as used here, which might allow refined testing of phonological vs. phonetic levels of representation. CVC syllables were used to allow comparisons of classifiers trained on the same consonants in different serial positions. We expect using the more common CV frame would produce similar results, but varying syllable types might permit additional testing related to the representation of abstract frames.

A substantial challenge in this and related work is the problem of disentangling input- and output-linked responses during simple repetition, when the stimulus and vocal production have the same class. This leads to difficulty, for instance, in interpreting if an input-linked 
response in frontal cortex is important for perception or if it simply reflects preparation of the sound for production. Work is currently ongoing to address this confound using a design that sometimes breaks the symmetry between input and output (i.e., the subject produces a different syllable or word than the one heard; see also Cogan et al., 2014).

Finally, it is important to note that although we focused our analyses on the cerebral cortex, the cerebellum and other subcortical structures are likely to also play roles in the representation of speech sounds and warrant further investigation. MVPA may be applied volumetrically to probe these areas, though it is critical to constrain searchlights anatomically (as was done here for the cortex) to ensure interpretability of the roles of individual areas / nuclei.

\section{Conclusion}

Repetition of a speech sound requires a speaker to engage a sequence of encoding and maintenance processes, invoking both auditory and motor representations of the sound. In this study, we sought to capture BOLD responses to speech items at distinct input and output stages of a simple syllable repetition task, and to use multivariate analysis to discover informational correlates of the speech items.

Our approach utilized a fast, event-related design, which allowed us to separately estimate input- and output-related BOLD responses. These individual trial response estimates allowed for the localization of clusters of cortical vertices that, across subjects, predicted the sounds heard or produced at above chance levels. It is also worth noting that, in general, stronger classification accuracies were observed in output-related analyses; whether this can be attributed to physiological differences at early and late stages of each trial or whether this indicates that output-related signals were more discernible in our protocol (perhaps due to detailed timing of scan acquisitions) remains unclear.

MVPA analyses revealed informational correlates of speech items predominantly in areas traditionally associated with the speech network (superior temporal, inferior frontal, preSMA, motor and somatosensory cortices). Of particular interest, the perceived vowel (the most acoustically salient sound available on a trial) could be predicted at above chance levels in the left inferior frontal sulcus, an area suggested previously to code for individual planned phonemes (Bohland and Guenther, 2006; Bohland et al., 2010). Likewise, the produced vowel could be detected from output-related responses in bilateral posterior STs, which indicate a target sound representation for production that is activated following the stimulus input, or auditory input of the speaker's own voice used for speech monitoring. Efforts to disentangle phonological (categorical) representations from phonetic (continuous) representations were limited in their utility, failing to reveal any significant dependence of classifiers on responses linearly related to formant frequencies. Further work is needed to clarify whether representations are categorical or track continuous acoustic features and to more completely dissociate the representations tied to speech perception and production. 
2 This research was supported by the Center of Excellence for Learning in Education, 3 Science, and Technology, a National Science Foundation Science of Learning Center (NSF 4 SMA-0835976, PI Shinn-Cunningham). The authors would like to thank Jacqueline You, 5 Jason Tourville, Frank Guenther, Mohammad Moghadamfalahi, Murat Akcakaya and Deniz 6 Erdogmus for their assistance and discussions of this work. 
Abrams, D.A., Ryali, S., Chen, T., Balaban, E., Levitin, D.J., Menon, V., 2013. Multivariate activation and connectivity patterns discriminate speech intelligibility in Wernicke's, Broca's, and Geschwind's areas. Cerebral Cortex 23, 1703-1714. doi 10.1093/cercor/bhs165.

Adank, P., 2012. The neural bases of difficult speech comprehension and speech production: Two Activation Likelihood Estimation (ALE) meta-analyses. Brain and Language 122, 42-54. doi 10.1016/j.bandl. 2012.04.014

Alario, F.X., Chainay, H., Lehericy, S., Cohen, L., 2006. The role of the supplementary motor area (SMA) in word production. Brain Research 1076, 129-143. doi 10.1016/j.brainres.2005.11.104.

Arsenault, J.S., Buchsbaum, B.R., 2015. Distributed Neural Representations of Phonological Features during Speech Perception. The Journal of Neuroscience 35, 634-642. doi:10.1523/JNEUROSCI.2454-14.2015.

Awh, E., Jonides, J., Smith, E.E., Schumacher, E.H., Koeppe, R.A., Katz, S., 1996. Dissociation of storage and rehearsal in verbal working memory: Evidence from positron emission tomography. Psychological Science 7, 25-31.

Baayen, R.H., Piepenbrock, R., Rijn, v., 1993. The CELEX lexical data base on CD-ROM. Linguistic Data Consortium, Philadelphia, PA.

Baldo, J.V., Katseff, S., Dronkers, N.F., 2012. Brain Regions Underlying Repetition and Auditory-Verbal Short-term Memory Deficits in Aphasia: Evidence from Voxel-based Lesion Symptom Mapping. Aphasiology 26, 338-354. doi $10.1080 / 02687038.2011 .602391$.

Binder, J.R., Frost, J.A., Hammeke, T.A., Rao, S., Cox, R.W., 1996. Function of the left planum temporale in auditory and linguisitic production. Brain 119, 1239-1247.

Boersma, P., Weenink, D., . Praat: doing phonetics by computer [Computer program]. Version 5.3.16, retrieved May 12, 2012, from http://www.praat.org/.

Bohland, J.W., Bullock, D., Guenther, F.H., 2010. Neural representations and mechanisms for the performance of simple speech sequences. Journal of Cognitive Neuroscience 22, 1504-1529. doi:10.1162/jocn. 2009.21306 .

Bohland, J.W., Guenther, F.H., 2006. An fMRI investigation of syllable sequence production. NeuroImage 32, 821-841. doi $10.1016 / \mathrm{j}$.neuroimage.2006.04.173.

Bonte, M., Hausfeld, L., Scharke, W., Valente, G., Formisano, E., 2014. Task-dependent decoding of speaker and vowel identity from auditory cortical response patterns. The Journal of Neuroscience 34, 4548-57. doi $10.1523 /$ JNEUROSCI . 4339-13.2014

Britton, B., Blumstein, S.E., Myers, E.B., Grindrod, C., 2009. The role of spectral and durational properties on hemispheric asymmetries in vowel perception. Neuropsychologia 47, 1096-1106. doi:10.1016/j. neuropsychologia.2008.12.033

Buchsbaum, B.R., Baldo, J., Okada, K., Berman, K.F., Dronkers, N., D’Esposito, M., Hickok, G., 2011. Conduction aphasia, sensory-motor integration, and phonological short-term memory An aggregate analysis of lesion and fMRI data. Brain and Language 119, 119-128. doi 10.1016/j.bandl.2010.12.001.

Carreiras, M., Mechelli, A., Price, C.J., 2006. Effect of word and syllable frequency on activation during lexical decision and reading aloud. Human Brain Mapping 27, 963-972. doi $10.1002 / \mathrm{hbm} .20236$.

Carreiras, M., Riba, J., Vergara, M., Heldmann, M., Münte, T.F., 2009. Syllable congruency and word frequency effects on brain activation. Human Brain Mapping 30, 3079-3088. doi 10.1002/hbm.20730.

Caspers, S., Geyer, S., Schleicher, A., Mohlberg, H., Amunts, K., Zilles, K., 2006. The human inferior parietal cortex: Cytoarchitectonic parcellation and interindividual variability. NeuroImage 33, 430-448. doi $10.1016 / \mathrm{j}$.neuroimage.2006.06.054

Catani, M., Jones, D.K., Ffytche, D.H., 2005. Perisylvian language networks of the human brain. Annals of Neurology 57, 8-16. doi $10.1002 /$ ana.20319

Celsis, P., Boulanouar, K., Doyon, B., Ranjeva, J.P., Berry, I., Nespoulous, J.L., Chollet, F., 1999. Differential fMRI responses in the left posterior superior temporal gyrus and left supramarginal gyrus to habituation and change detection in syllables and tones. NeuroImage 9, 135-144. doi:10.1006/nimg.1998.0389.

Chen, Y., Namburi, P., Elliott, L.T., Heinzle, J., Soon, C.S., Chee, M.W.L., Haynes, J.D., 2011. Cortical surface-based searchlight decoding. NeuroImage 56, 582-592. doi $10.1016 / j$.neuroimage 2010.07 .035 .

Cogan, G.B., Thesen, T., Carlson, C., Doyle, W., Devinsky, O., Pesaran, B., 2014. Sensory-motor transfor- 
mations for speech occur bilaterally. Nature 507, 94-98. doi:10.1038/nature12935

Corfield, D.R., Murphy, K., Josephs, O., Fink, G.R., Frackowiak, R.S.J., Guz, A., Adams, L., Turner, R., 1999. Cortical and subcortical control of tongue movement in humans: a functional neuroimaging study using fMRI. Journal of applied physiology (Bethesda, Md. : 1985) 86, 1468-1477.

Correia, J.M., Jansma, B.M.B., Bonte, M., 2015. Decoding Articulatory Features from fMRI Responses in Dorsal Speech Regions. The Journal of Neuroscience 35, 15015-15025. doi:10.1523/JNEUROSCI.0977-15. 2015 .

Dale, A.M., Fischl, B., Sereno, M.I., 1999. Cortical Surface-Based Analysis. NeuroImage 194, 179-194.

D'Ausilio, A., Pulvermüller, F., Salmas, P., Bufalari, I., Begliomini, C., Fadiga, L., 2009. The Motor Somatotopy of Speech Perception. Current Biology 19, 381-385. doi: $10.1016 / j$.cub.2009.01.017.

Davis, T., LaRocque, K.F., Mumford, J.A., Norman, K.A., Wagner, A.D., Poldrack, R.A., 2014. What do differences between multi-voxel and univariate analysis mean? How subject-, voxel-, and trial-level variance impact fMRI analysis. NeuroImage 97, 271-283. doi $10.1016 / j$.neuroimage .2014.04.037.

Davis, T., Poldrack, R.A., 2013. Measuring neural representations with fMRI: Practices and pitfalls. Annals of the New York Academy of Sciences 1296, 108-134. doi 10.1111/nyas.12156.

Dell, G.S., Burger, L.K., Svec, W.R., 1997. Language production and serial order: a functional analysis and a model. Psychological Review 104, 123-47. doi:10.1037/0033-295X.104.1.123.

Deschamps, I., Tremblay, P., 2014. Sequencing at the syllabic and supra-syllabic levels during speech perception: an fMRI study. Frontiers in Human Neuroscience 8, 1-14. doi 10.3389/fnhum.2014.00492.

Desikan R. S. et al., 2006. An automated labeling system for subdividing the human cerebral cortex on MRI scans into gyral based regions of interest. NeuroImage 31, 968-980. doi:10.1016/j.neuroimage. 2006 . 01.021 .

Dijkstra, E.W., 1959. A note on two problems in connexion with graphs. Numerische Mathematik 1, 269-271. doi 10.1007/BF01386390.

Du, Y., Buchsbaum, B.R., Grady, C.L., Alain, C., 2014. Noise differentially impacts phoneme representations in the auditory and speech motor systems. Proceedings of the National Academy of Sciences of the United States of America 111, 7126-31. doi $10.1073 /$ pnas.1318738111.

Eden, G.F., Joseph, J.E., Brown, H.E., Brown, C.P., Zeffiro, T.A., 1999. Utilizing hemodynamic delay and dispersion to detect fMRI signal change without auditory interference: The behavior interleaved gradients technique. Magnetic Resonance in Medicine 41, 13-20. doi:10.1002/(SICI)1522-2594(199901)41: 1<13: :AID-MRM4>3.0.CO;2-T

Edmister, W.B., Talavage, T.M., Ledden, P.J., Weisskoff, R.M., 1999. Improved auditory cortex imaging using clustered volume acquisitions. Human Brain Mapping 7, 89-97. doi 10.1002/(SICI) 1097-0193(1999) $7: 2<89:$ :AID-HBM2>3.0.CO;2-N.

Etzel, J.A., Zacks, J.M., Braver, T.S., 2013. Searchlight analysis: Promise, pitfalls, and potential. NeuroImage 78, 261-269. doi $10.1016 / \mathrm{j}$.neuroimage.2013.03.041.

Evans, S., Davis, M.H., 2015. Hierarchical Organization of Auditory and Motor Representations in Speech Perception: Evidence from Searchlight Similarity Analysis. Cerebral Cortex 25, 4772-88. doi 10.1093/ cercor/bhv136.

Fadiga, L., Craighero, L., 2003. New insights on sensorimotor integration: From hand action to speech perception. Brain and Cognition 53, 514-524. doi 10.1016/S0278-2626(03)00212-4.

Fiez, J.A., Raife, E.A., Balota, D.A., Schwarz, J.P., Raichle, M.E., Petersen, S.E., 1996. A positron emission tomography study of the short-term maintenance of verbal information. The Journal of Neuroscience 16, 808-822.

Fischl, B., 2012. FreeSurfer. NeuroImage 62, 774-781. doi:10.1016/j.neuroimage.2012.01.021.

Flinker, A., Chang, E.F., Kirsch, H.E., Barbaro, N.M., Crone, N.E., Knight, R.T., 2010. Single-trial speech suppression of auditory cortex activity in humans. The Journal of Neuroscience 30, 16643-16650. doi 10.1523 /JNEUROSCI . 1809-10.2010.

Formisano, E., De Martino, F., Bonte, M., Goebel, R., 2008. "Who" is saying "what"? Brain-based decoding of human voice and speech. Science 322, 970-973. doi $10.1126 /$ science.1164318.

Friston, K.J., Williams, S., Howard, R., Frackowiak, R.S.J., Turner, R., 1996. Movement-related effects in 
fMRI time-series. Magnetic Resonance in Medicine 35, 346-355. doi:10.1002/mrm.1910350312.

Gathercole, S.E., 1995. Is nonword repetition a test of phonological memory or long-term knowledge? It all depends on the nonwords. Memory \\& Cognition 23, 83-94. doi 10.3758/BF03210559.

Goldrick, M., Rapp, B., 2007. Lexical and post-lexical phonological representations in spoken production. Cognition 102, 219-260. doi 10.1016/j.cognition.2005.12.010.

Graves, W.W., Grabowski, T.J., Mehta, S., Gupta, P., 2008. The left posterior superior temporal gyrus participates specifically in accessing lexical phonology. Journal of cognitive neuroscience 20, 1698-1710. doi:10.1162/jocn.2008.20113.

Guenther, F.H., Ghosh, S.S., Tourville, J.A., 2006. Neural modeling and imaging of the cortical interactions underlying syllable production. Brain and Language 96, 280-301. doi:10.1016/j.bandl.2005.06.001. arXiv:NIHMS150003.

Hall, D.A., Haggard, M.P., Akeroyd, M.A., Palmer, A.R., Summerfield, A.Q., Elliott, M.R., Gurney, E.M., Bowtell, R.W., 1999. 'Sparse' temporal sampling in auditory fMRI. Human Brain Mapping 7, $213-223$. doi 10.1002/(SICI) 1097-0193(1999) $7: 3<213::$ AID-HBM5>3.0.CO;2-N.

Hanke, M., Halchenko, Y.O., Sederberg, P.B., Hanson, S.J., Haxby, J.V., Pollmann, S., 2009. PyMVPA: A python toolbox for multivariate pattern analysis of fMRI data. Neuroinformatics 7, 37-53. doi:10.1007/ s12021-008-9041-y.

Hanley, J.R., Dell, G., Kay, J., Baron, R., 2004. Evidence for the involvement of a nonlexical route in the repetition of familiar words: A comparison of single and dual route models of auditory repetition. Cognitive Neuropsychology 21, 147-158. doi 10.1080/02643290342000339.

Hartley, T., Houghton, G., 1996. A linguistically constrained model of short-term memory for nonwords. Journal of Memory and Language 35, 1-31. doi $10.1006 / \mathrm{jmla} .1996 .0001$

Haxby, J.V., Gobbini, M.I., Furey, M.L., Ishai, A., Schouten, J.L., Pietrini, P., 2001. Distributed and overlapping representations of faces and objects in ventral temporal cortex. Science 293, 2425-2430. doi:10.1126/science.1063736.

Hester, R., D'Esposito, M., Cole, M.W., Garavan, H., 2007. Neural mechanisms for response selection: comparing selection of responses and items from working memory. NeuroImage 34, 446-454. doi 10 . 1016/j.neuroimage.2006.08.001.

Hickok, G., Erhard, P., Kassubek, J., Helms-Tillery, A.K., Naeve-Velguth, S., Strupp, J.P., Strick, P.L., Ugurbil, K., 2000. A functional magnetic resonance imaging study of the role of left posterior superior temporal gyrus in speech production: implications for the explanation of conduction aphasia. Neuroscience letters 287, 156-160.

Hickok, G., Houde, J., Rong, F., 2011. Sensorimotor integration in speech processing: computational basis and neural organization. Neuron 69, 407-22. doi 10.1016/j.neuron.2011.01.019.

Hickok, G., Poeppel, D., 2000. Towards a functional neuroanatomy of speech perception. Trends in Cognitive Sciences 4, 131-138. doi 10740277.

Hickok, G., Poeppel, D., 2004. Dorsal and ventral streams: A framework for understanding aspects of the functional anatomy of language. Cognition 92, 67-99. doi:10.1016/j.cognition.2003.10.011.

Hickok, G., Poeppel, D., 2007. The cortical organization of speech processing. Nature reviews. Neuroscience 8, 393-402. doi:10.1038/nrn2113.

Houde, J.F., Nagarajan, S.S., Sekihara, K., Merzenich, M.M., 2002. Modulation of the auditory cortex during speech: an MEG study. Journal of Cognitive Neuroscience 14, 1125-1138. doi 10.1162/ 089892902760807140

Hynd, G.W., Hynd, C.R., 1984. Dyslexia : Neuroanatomical / neurolinguistic perspectives. Reading Research Quarterly 19, 482-498.

Jacquemot, C., Dupoux, E., Bachoud-Lévi, A.C., 2007. Breaking the mirror: Asymmetrical disconnection between the phonological input and output codes. doi:10.1080/02643290600683342.

Jefferies, E., Crisp, J., Ralph, M.A.L., 2006. The impact of phonological or semantic impairment on delayed auditory repetition: Evidence from stroke aphasia and semantic dementia. Aphasiology 20, 963-992. doi $10.1080 / 02687030600739398$.

Jonas, S., 1981. The supplementary motor region and speech emission. Journal of Communication Disorders 
14, 349-373. doi 10.1016/0021-9924(81)90019-8.

Jonides, J., Schumacher, E.H., Smith, E.E., Koeppe, R.A., Awh, E., Reuter-Lorenz, P.A., Marshuetz, C., Willis, C.R., 1998. The role of parietal cortex in verbal working memory. Journal of Neuroscience 18, 5026-5034.

Kilian-Hütten, N., Valente, G., Vroomen, J., Formisano, E., 2011. Auditory cortex encodes the perceptual interpretation of ambiguous sound. The Journal of Neuroscience 31, 1715-20. doi $10.1523 /$ JNEUROSCI . 4572-10.2011.

Kriegeskorte, N., Goebel, R., Bandettini, P., 2006. Information-based functional brain mapping. Proceedings of the National Academy of Sciences of the United States of America 103, 3863-3868. doi:10.1073/pnas . 0600244103 .

Kriegeskorte, N., Mur, M., Bandettini, P., 2008. Representational similarity analysis - connecting the branches of systems neuroscience. Frontiers in Systems Neuroscience 2, 4. doi $10.3389 /$ neuro.06.004. 2008.

Lee, Y.S., Turkeltaub, P., Granger, R., Raizada, R.D.S., 2012. Categorical Speech Processing in Broca's Area: An fMRI Study Using Multivariate Pattern-Based Analysis. Journal of Neuroscience 32, 3942-3948. doi $10.1523 /$ JNEUROSCI . 3814-11.2012.

Levelt, W.J.M., Wheeldon, L., 1994. Do speakers have access to a mental syllabary? Cognition 50, $239-269$. doi $10.1016 / 0010-0277(94) 90030-2$.

Liberman, A.M., Cooper, F.S., Shankweiler, D.P., Studdert-Kennedy, M., 1967. Perception of the speech code. Psychological Review 74, 431-461. doi:10.1037/h0020279.

Lichtheim, L., 1885. On Aphasia. Brain 7, 433-484. doi:10.1093/brain/7.4.433.

Lotze, M., Seggewies, G., Erb, M., Grodd, W., Birbaumer, N., 2000. The representation of articulation in the primary sensorimotor cortex. Neuroreport 11, 2985-2989. doi:10.1097/00001756-200009110-00032.

MacNeilage, P.F., 1998. The frame/content theory of evolution of speech production. Behavioral and Brain Sciences 21, 499-511. doi:10.1017/S0140525X98001265

Markiewicz, C.J., 2016. philips-cdas v0.1. doi:10.5281/zenodo.49853.

Martin, R.C., Breedin, S.D., Damian, M.F., 1999. The relation of phoneme discrimination, lexical access, and short-term memory: A case study and interactive activation account. Brain and Language 70, 437-482. doi $10.1006 /$ brln.1999.2184.

Martuzzi, R., Ramani, R., Qiu, M., Rajeevan, N., Constable, R.T., 2010. Functional connectivity and alterations in baseline brain state in humans. NeuroImage 49, 823-834. doi $10.1016 / \mathrm{j}$. neuroimage. 2009.07 .028 .

McGettigan, C., Warren, J.E., Eisner, F., Marshall, C.R., Shanmugalingam, P., Scott, S.K., 2011. Neural correlates of sublexical processing in phonological working memory. Journal of Cognitive Neuroscience 23, 1-17. doi:10.1162/jocn.2010.21491.

Mei L. et al., 2014. Artificial language training reveals the neural substrates underlying addressed and assembled phonologies. PLoS ONE 9, e93548. doi 10.1371/journal.pone.0093548.

Meister, I.G., Wilson, S.M., Deblieck, C., Wu, A.D., Iacoboni, M., 2007. The Essential Role of Premotor Cortex in Speech Perception. Current Biology 17, 1692-1696. doi:10.1016/j.cub.2007.08.064.

Menenti, L., Segaert, K., Hagoort, P., 2012. The neuronal infrastructure of speaking. Brain and Language 122, 71-80. doi:10.1016/j.bandl.2012.04.012.

Merrill, J., Sammler, D., Bangert, M., Goldhahn, D., Lohmann, G., Turner, R., Friederici, A.D., 2012. Perception of words and pitch patterns in song and speech. Frontiers in Psychology 3, 1-13. doi 10 . 3389/fpsyg. 2012.00076.

Molnar-Szakacs, I., Iacoboni, M., Koski, L., Mazziotta, J.C., 2005. Functional Segregation within Pars Opercularis of the Inferior Frontal Gyrus: Evidence from fMRI Studies of Imitation and Action Observation. Cerebral Cortex 15, 986-994. doi:10.1093/cercor/bhh199.

Mumford, J.A., Turner, B.O., Ashby, F.G., Poldrack, R.A., 2012. Deconvolving BOLD activation in eventrelated designs for multivoxel pattern classification analyses. NeuroImage 59, 2636-2643. doi:10.1016/ j.neuroimage.2011.08.076.

Myers, E.B., 2007. Dissociable effects of phonetic competition and category typicality in a pho- 
netic categorization task: An fMRI investigation. Neuropsychologia 45, 1463-1473. doi:10.1016/j. neuropsychologia.2006.11.005

Myers, E.B., Blumstein, S.E., Walsh, E., Eliassen, J., 2009. Inferior frontal regions underlie the perception of phonetic category invariance. Psychological Science 20, 895-903. doi $10.1111 / j .1467-9280.2009$. $02380 . x$.

Norman, K.A., Polyn, S.M., Detre, G.J., Haxby, J.V., 2006. Beyond mind-reading: multi-voxel pattern analysis of fMRI data. Trends in Cognitive Sciences 10, 424-430. doi:10.1016/j.tics.2006.07.005.

Nozari, N., Dell, G.S., 2013. How damaged brains repeat words: A computational approach. Brain and Language 126, 327-337. doi $10.1016 / \mathrm{j}$.bandl.2013.07.005

Numminen, J., Salmelin, R., Hari, R., 1999. Subject's own speech reduces reactivity of the human auditory cortex. Neuroscience Letters 265, 119-122. doi 10.1016/S0304-3940(99)00218-9.

Okada, K., Hickok, G., 2006. Left posterior auditory-related cortices participate both in speech perception and speech production: Neural overlap revealed by fMRI. Brain and Language 98, 112-117. doi 10 . 1016/j. bandl.2006.04.006

Okada, K., Smith, K.R., Humphries, C., Hickok, G., 2003. Word length modulates neural activity in auditory cortex during covert object naming. Neuroreport 14. doi:10.1097/01. wnr.0000094104.16607.

Oosterhof, N.N., Wiestler, T., Downing, P.E., Diedrichsen, J., 2011. A comparison of volume-based and surface-based multi-voxel pattern analysis. NeuroImage 56, 593-600. doi:10.1016/j.neuroimage. 2010 . 04.270 .

Papoutsi, M., de Zwart, J.A., Jansma, J.M., Pickering, M.J., Bednar, J.A., Horwitz, B., 2009. From phonemes to articulatory codes: An fMRI study of the role of Broca's area in speech production. Cerebral Cortex 19, 2156-2165. doi $10.1093 /$ cercor/bhn239

Paulesu, E., Frith, C.D., Frackowiak, R.S., 1993. The neural correlates of the verbal component of working memory. Nature $362,342-345$. doi $10.1038 / 362342 \mathrm{a} 0$

Paus, T., 1996. Modulation of cerebral blood flow in the human auditory cortex during speech: Role of motorto-sensory discharges. European Journal of Neuroscience 8, 2236-2246. doi 10.1111/j.1460-9568.1996. tb01187.x

Peeva, M.G., Guenther, F.H., Tourville, J.A., Nieto-Castanon, A., Anton, J.L., Nazarian, B., Alario, F.X., 2010. Distinct representations of phonemes, syllables, and supra-syllabic sequences in the speech production network. NeuroImage 50,626-638. doi $10.1016 / j$. neuroimage .2009 .12 .065 .

Perrachione, T.K., Ghosh, S.S., 2013. Optimized design and analysis of sparse-sampling fMRI experiments. Frontiers in Neuroscience 7, 55. doi $10.3389 /$ fnins.2013.00055

Peterson, G.E., Barney, H.H., 1952. Control methods used in a study of the vowels. Journal of the Acoustical Society of America 24, 175-184.

Price, C.J., 2012. A review and synthesis of the first 20years of PET and fMRI studies of heard speech, spoken language and reading. NeuroImage 62, 816-847. doi $10.1016 / j$. neuroimage.2012.04.062

Pugh, K.R., Mencl, W.E., Jenner, A.R., Katz, L., Frost, S.J., Lee, J.R., Shaywitz, S.E., Shaywitz, B.A., 2001. Neurobiological studies of reading and reading disability. Journal of Communication Disorders 34, 479-492. doi 10.1016/S0021-9924(01)00060-0.

Pulvermüller, F., Huss, M., Kherif, F., Moscoso del Prado Martin, F., Hauk, O., Shtyrov, Y., 2006. Motor cortex maps articulatory features of speech sounds. Proceedings of the National Academy of Sciences of the United States of America 103, 7865-7870. doi:10.1073/pnas.0509989103.

Raizada, R.D.S., Poldrack, R.A., 2007. Selective Amplification of Stimulus Differences during Categorical Processing of Speech. Neuron 56, 726-740. doi 10.1016/j.neuron.2007.11.001.

Rauschecker, J.P., Scott, S.K., 2009. Maps and streams in the auditory cortex: nonhuman primates illuminate human speech processing. Nature neuroscience 12, 718-724. doi:10.1038/nn.2331.

Ravizza, S.M., Delgado, M.R., Chein, J.M., Becker, J.T., Fiez, J.A., 2004. Functional dissociations within the inferior parietal cortex in verbal working memory. NeuroImage $22,562-73$. doi $10.1016 / \mathrm{j}$.neuroimage. 2004.01 .039 .

Riecker, A., Ackermann, H., Wildgruber, D., Dogil, G., Grodd, W., 2000. Opposite hemispheric lateralization effects during speaking and singing at motor cortex, insula and cerebellum. Neuroreport 11, 1997-2000. 
doi $10.1097 / 00001756-200006260-00038$

Roelofs, A., 1997. The WEAVER model of word-form encoding in speech production. Cognition 64, $249-284$. doi 10.1016/S0010-0277(97)00027-9.

Rogalsky, C., Poppa, T., Chen, K.H., Anderson, S.W., Damasio, H., Love, T., Hickok, G., 2015. Speech repetition as a window on the neurobiology of auditory-motor integration for speech: A voxel-based lesion symptom mapping study. Neuropsychologia 71, 18-27. doi 10.1016/j .neuropsychologia.2015.03.012.

Rottschy, C., Langner, R., Dogan, I., Reetz, K., Laird, A.R., Schulz, J.B., Fox, P.T., Eickhoff, S.B., 2012. Modelling neural correlates of working memory: A coordinate-based meta-analysis. NeuroImage 60, 830-846. doi $10.1016 / \mathrm{j} \cdot$ neuroimage.2011.11.050

Saur D. et al., 2008. Ventral and dorsal pathways for language. Proceedings of the National Academy of Sciences 105, 18035-18040. doi 10.1073/pnas.0805234105

Schmidt, C.F., Zaehle, T., Meyer, M., Geiser, E., Boesiger, P., Jancke, L., 2008. Silent and continuous fMRI scanning differentially modulate activation in an auditory language comprehension task. Human Brain Mapping 29, 46-56. doi $10.1002 / \mathrm{hbm} .20372$.

Stelzer, J., Chen, Y., Turner, R., 2013. Statistical inference and multiple testing correction in classificationbased multi-voxel pattern analysis (MVPA): Random permutations and cluster size control. NeuroImage 65, 69-82. doi $10.1016 / j$. neuroimage.2012.09.063

Todd, M.T., Nystrom, L.E., Cohen, J.D., 2013. Confounds in multivariate pattern analysis: Theory and rule representation case study. NeuroImage 77, 157-165. doi:10.1016/j.neuroimage.2013.03.039.

Tomasi, D., Goldstein, R.Z., Telang, F., Maloney, T., Alia-Klein, N., Caparelli, E.C., Volkow, N.D., 2007. Widespread disruption in brain activation patterns to a working memory task during cocaine abstinence. Brain Research 1171, 83-92. doi:10.1016/j.brainres.2007.06.102, arXiv:NIHMS150003.

Tourville, J.A., Guenther, F.H., 2012. Automatic cortical labeling system for neuroimaging studies of normal and disordered speech, in: Neuroscience Meeting Planner, Society for Neuroscience, New Orleans, LA. p. 681.06 .

Tourville, J.A., Reilly, K.J., Guenther, F.H., 2008. Neural mechanisms underlying auditory feedback control of speech. NeuroImage 39, 1429-1443. doi 10.1016/j.neuroimage.2007.09.054, arXiv:NIHMS150003.

Tremblay, P., Baroni, M., Hasson, U., 2013a. Processing of speech and non-speech sounds in the supratemporal plane: Auditory input preference does not predict sensitivity to statistical structure. NeuroImage 66, 318-332. doi $10.1016 / j . n e u r o i m a g e .2012 .10 .055$.

Tremblay, P., Deschamps, I., Gracco, V.L., 2013b. Regional heterogeneity in the processing and the production of speech in the human planum temporale. Cortex 49, 143-157. doi:10.1016/j.cortex.2011.09.004.

Tremblay, P., Gracco, V.L., 2006. Contribution of the frontal lobe to externally and internally specified verbal responses: fMRI evidence. NeuroImage 33, 947-957. doi:10.1016/j.neuroimage.2006.07.041.

Tremblay, P., Gracco, V.L., 2009. Contribution of the pre-SMA to the production of words and non-speech oral motor gestures, as revealed by repetitive transcranial magnetic stimulation (rTMS). Brain Research 1268, 112-124. doi:10.1016/j.brainres.2009.02.076.

Tremblay, P., Gracco, V.L., 2010. On the selection of words and oral motor responses: Evidence of a response-independent fronto-parietal network. Cortex 46, 15-28. doi $10.1016 /$ j.cortex.2009.03.003.

Tremblay, P., Small, S.L., 2011. Motor response selection in overt sentence production: a functional MRI study. Frontiers in Psychology 2, 1-14. doi $10.3389 /$ fpsyg.2011.00253.

Vaden, K.I., Muftuler, L.T., Hickok, G., 2010. Phonological repetition-suppression in bilateral superior temporal sulci. NeuroImage 49, 1018-1023. doi 10.1016/j.neuroimage.2009.07.063

Wildgruber, D., Ackermann, H., Klose, U., Kardatzki, B., Grodd, W., 1996. Functional lateralization of speech production at primary motor cortex: a fMRI study. Neuroreport 7, 2791-2795.

Wilson, S.M., Saygin, A.P., Sereno, M.I., Iacoboni, M., 2004. Listening to speech activates motor areas involved in speech production. Nature Neuroscience 7, 701-2. doi 10.1038/nn1263.

Wise, R.J.S., Scott, S.K., Blank, S.C., Mummery, C.J., Murphy, K., Warburton, E.A., 2001. Separate neural subsystems within 'Wernicke's area'. Brain 124, 83-95. doi 10.1093/brain/124.1.83.

You, J., Markiewicz, C.J., Bohland, J.W., 2015. Formant detection scripts for "mapping the cortical representation of speech sounds in a syllable repetition task. doi: $10.5281 /$ zenodo. 51362 . 
Zaehle, T., Schmidt, C.F., Meyer, M., Baumann, S., Baltes, C., Boesiger, P., Jancke, L., 2007. Comparison of "silent" clustered and sparse temporal fMRI acquisitions in tonal and speech perception tasks. NeuroImage 37, 1195-1204. doi 10.1016/j.neuroimage.2007.04.073.

Zhang, Q., Hu, X., Luo, H., Li, J., Zhang, X., Zhang, B., 2016. Deciphering phonemes from syllables in BOLD signals in human superior temporal gyrus. European Journal of Neuroscience 43, 773-781. doi $10.1111 /$ ejn.13164.

Ziegler, W., Kilian, B., Deger, K., 1997. The role of the left mesial frontal cortex in fluent speech: Evidence from a case of left supplementary motor area hemorrhage. Neuropsychologia 35, 1197-1208. doi 10.1016/ S0028-3932(97) 00040-7. 